\title{
RIPK3 promotes sepsis-induced acute kidney injury via mitochondrial dysfunction
}

\author{
Angara Sureshbabu, ${ }^{1}$ Edwin Patino, ${ }^{2}$ Kevin C. Ma, ${ }^{1}$ Kristian Laursen, ${ }^{3}$ Eli J. Finkelsztein, ${ }^{1}$ \\ Oleh Akchurin, ${ }^{4}$ Thangamani Muthukumar, ${ }^{2,5}$ Stefan W. Ryter, ${ }^{1}$ Lorraine Gudas, ${ }^{3}$ \\ Augustine M. K. Choi, ${ }^{1,5}$ and Mary E. Choi ${ }^{2,5}$ \\ 'Division of Pulmonary and Critical Care Medicine, and 'Division of Nephrology and Hypertension, Joan and Sanford I. Weill \\ Department of Medicine, Weill Cornell Medicine, New York, New York, USA. ${ }^{3}$ Department of Pharmacology, \\ ${ }^{4}$ Department of Pediatrics, and ${ }^{5}$ NewYork-Presbyterian Hospital, Weill Cornell Medical Center, New York, New York, USA.
}

\begin{abstract}
Sepsis causes acute kidney injury (AKI) in critically ill patients, although the pathophysiology remains unclear. The receptor-interacting protein kinase-3 (RIPK3), a cardinal regulator of necroptosis, has recently been implicated in the pathogenesis of human disease. In mice subjected to polymicrobial sepsis, we demonstrate that RIPK3 promotes sepsis-induced AKI. Utilizing genetic deletion and biochemical approaches in vitro and in vivo, we identify a potentially novel pathway by which RIPK3 aggravates kidney tubular injury independently of the classical mixed lineage kinase domain-like protein-dependent (MLKL-dependent) necroptosis pathway. In kidney tubular epithelial cells, we show that RIPK3 promotes oxidative stress and mitochondrial dysfunction involving upregulation of NADPH oxidase-4 (NOX4) and inhibition of mitochondrial complex I and -III, and that RIPK3 and NOX4 are critical for kidney tubular injury in vivo. Furthermore, we demonstrate that RIPK3 is required for increased mitochondrial translocation of NOX4 in response to proinflammatory stimuli, by a mechanism involving protein-protein interactions. Finally, we observed elevated urinary and plasma RIPK3 levels in human patients with sepsis-induced AKI, representing potential markers of this condition. In conclusion, we identify a pathway by which RIPK3 promotes kidney tubular injury via mitochondrial dysfunction, independently of MLKL, which may represent a promising therapeutic target in sepsis-induced AKI.
\end{abstract}

Conflict of interest: The authors have declared that no conflict of interest exists.

Submitted: October 31, 2017

Accepted: May 1, 2018

Published: June 7, 2018

Reference information: JCI Insight. 2018;3(11):e98411. https:// doi.org/10.1172/jci.insight.98411.

\section{Introduction}

Sepsis is a life-threatening organ dysfunction caused by a dysregulated host response to infection (1). The clinical management of sepsis includes the use of antimicrobial agents and fluid resuscitation, with recent improvements in supportive care such as hemodialysis, utilization of blood products, and management of nosocomial infections $(2,3)$. Sepsis most commonly results in multiorgan failure, including kidney failure as the result of acute kidney injury (AKI), leading to increased morbidity and mortality (4). The mechanisms underlying the pathophysiology of sepsis-induced AKI are complex and incompletely understood, but they may include disturbances in coagulation, inflammation, oxidative stress, and altered kidney tubular epithelial cell responses to injury (5)

Necroptosis, a form of regulated necrotic cell death with emerging significance in human disease in recent years, is regulated by signal transduction effector proteins, including RIPK1, RIPK3, and its downstream target MLKL (6). A number of cellular mediators, including death receptors, IFNs, TLRs, and RNA or DNA sensors, can activate the necroptosis pathway (7). Necroptosis, a form of cell death morphologically distinct from apoptosis, is characterized by the rupture of the plasma membrane, leakage of intracellular contents, and organelle swelling $(8,9)$.

Recent studies have demonstrated the upregulated expression of RIPK3 in murine models of renal ischemia/reperfusion injury and transfusion-associated endothelial cell injury $(10,11)$. Furthermore, elevated levels of RIPK3 have been reported in the plasma of trauma patients with AKI (12). However, the role of RIPK3 in sepsis-induced AKI has previously not been studied. We therefore sought to investigate the functional role of RIPK3 in experimental sepsis-induced AKI and, importantly, its relevance to human patients with AKI in sepsis. 
A

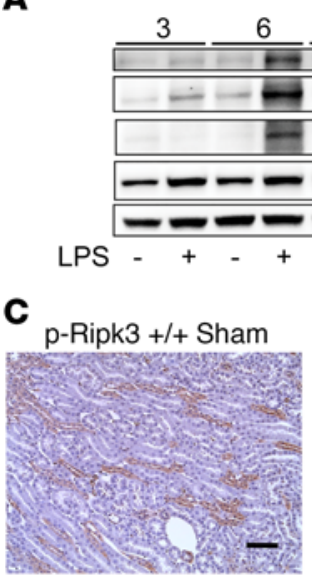

D Ripk3+1+ Sham

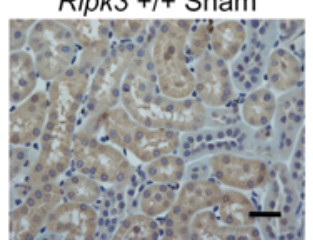

$12^{h}$ p-RIPK3 RIPK3 p-MLKL MLKL $\beta$-actin

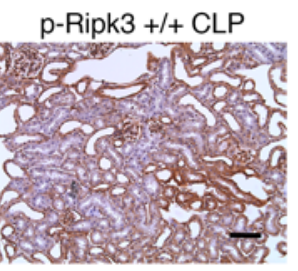

Ripk3 +/+ CLP

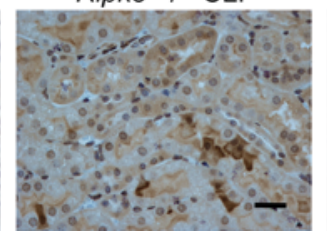

B

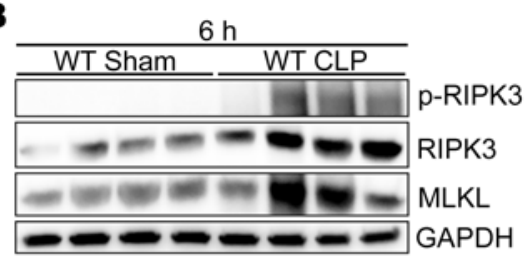

p-Ripk3 -/- Sham
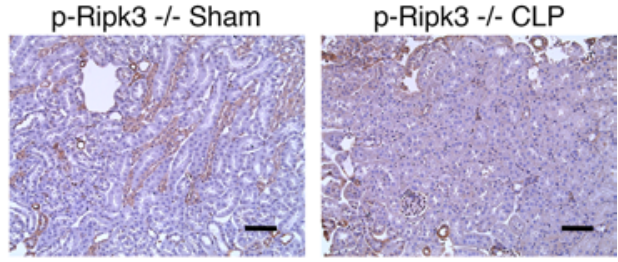

Ripk3 -/-Sham

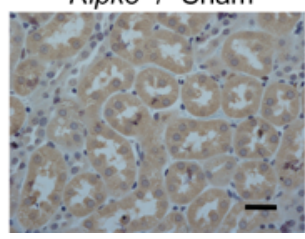

Ripk3 -I- CLP

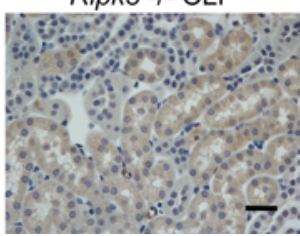

Figure 1. Sepsis increases RIPK3 expression in kidney tubular epithelial cells. (A) Western blot for the expression of p-RIPK3, RIPK3, and p-MLKL in HK-2 cells treated with or without LPS $(1 \mu \mathrm{g} / \mathrm{ml})$ for the indicated times. (B) Western blot for the expression of p-RIPK3, RIPK3, and MLKL in kidney homogenates of WT mice at 6 hours after CLP or sham surgery. Western blots shown are representative results from 3 independent experiments. (C and $\mathbf{D})$

Representative IHC images of RIPK3 (C) and RIPK3 (D) staining localized to the kidney tubular epithelial cells from Ripk $3^{+/+}$or Ripk $3^{-/-}$mice at 6 hours after CLP or sham surgery. ( $n=6$ for Sham; $n=6$ for CLP). Scale bars: $20 \mu \mathrm{m}$.

In the current study, we established RIPK3 as a crucial mediator of sepsis-induced AKI via a pathway that is independent of the classical RIPK3-activated MLKL-dependent necroptosis pathway. We further demonstrate that mitochondrial dysfunction, associated with altered regulation of NOX4 and mitochondrial complex I and -III in kidney tubular epithelial cells, represents a major underlying mechanism by which RIPK3 propagates experimental sepsis-induced AKI. In human studies, we identify elevated RIPK3 as a potential urinary and plasma marker of sepsis-induced AKI. Taken together, our findings suggest RIPK3 may serve as a therapeutic target in human sepsis-induced AKI.

\section{Results}

Sepsis increases the expression and activation of necroptosis proteins in kidney tubular epithelial cells. We first examined the expression of RIPK3 and the related necroptosis proteins RIPK1 and MLKL in sepsis-induced AKI. Since proximal tubular epithelial cells represent a primary target of sepsis, we utilized cultured human kidney proximal tubular epithelial cells (HK-2) challenged with bacterial LPS as an in vitro model of sepsis-associated kidney tubular injury. We also employed the cecal ligation and puncture (CLP) model, a widely accepted mouse model of polymicrobial sepsis.

The expression levels of phosphorylated RIPK3 (p-RIPK3), RIPK3, and p-MLKL, but not RIPK1, were time-dependently increased in HK-2 cells in response to LPS challenge, relative to untreated control cells (Figure 1A and Supplemental Figure 1A; supplemental material available online with this article; https://doi.org/10.1172/jci.insight.98411DS1). In mice subjected to CLP surgery, expression levels of p-RIPK3, RIPK3, and MLKL were also higher in the kidney tissues at 6 hours after treatment, relative to the sham-operated control mice (Figure 1B). Immunohistochemical analysis revealed increased expression levels of p-RIPK3 at 6 hours (Figure 1C) and RIPK3 at 6-12 hours (Figure 1D and Supplemental Figure 1B) in kidney tubular epithelial cells of WT $\left(\right.$ Ripk $\left.^{+/+}\right)$mice following CLP, relative to sham-operated mice.

Genetic deletion of RIPK3 suppresses kidney tubular injury and preserves kidney function. To determine the functional role of RIPK3 in sepsis-induced AKI, we subjected Ripk $3^{-/-}$mice, and their corresponding WT $R i p k 3^{+/+}$littermate mice, to CLP or sham surgery. At 24 hours following CLP surgery, markedly lower 
A

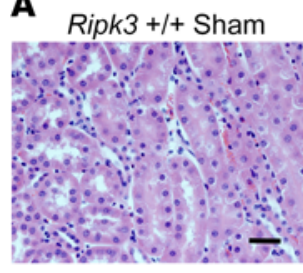

B
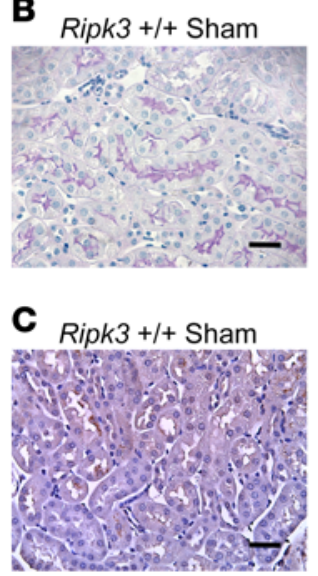

D

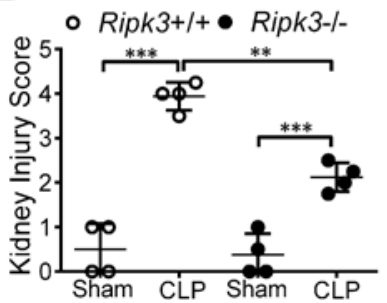

Ripk3 +/+ CLP

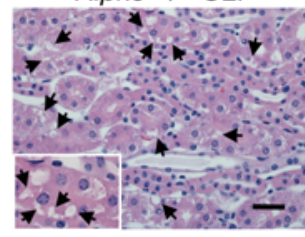

Ripk3 +1+ CLP

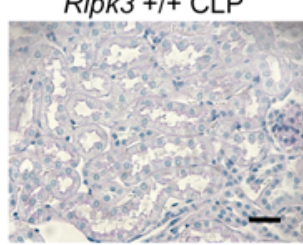

Ripk3 +/+ CLP

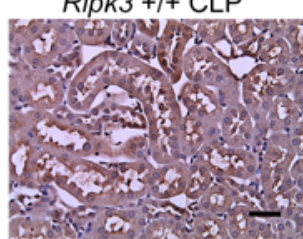

E

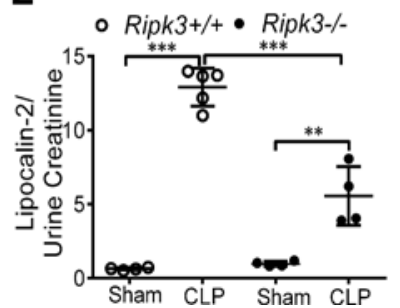

Ripk3 -/- Sham

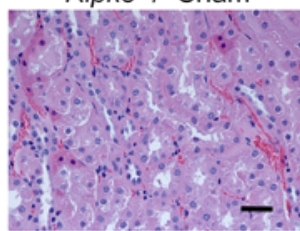

Ripk3 -/- Sham

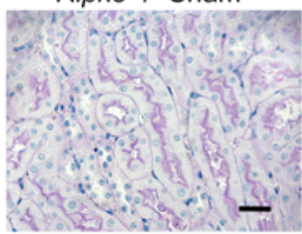

Ripk3 -/- Sham

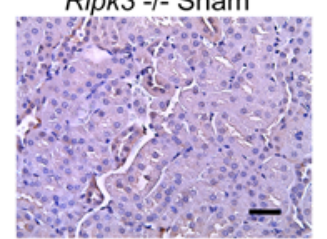

Ripk3 -/- CLP

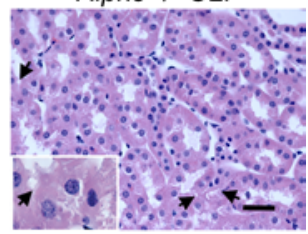

Ripk3 -/- CLP

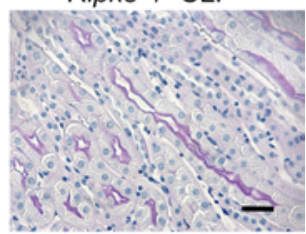

Ripk3-I- CLP

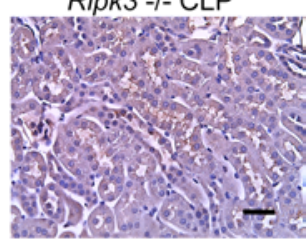

$\mathbf{F}$

Figure 2. Ripk3 deficiency protects against kidney tubular injury in sepsis. (A) Representative images of H\&E staining of tubular epithelial cells of kidney

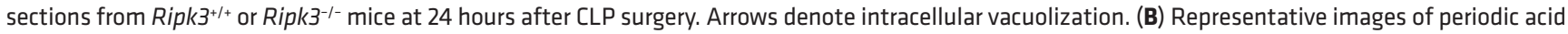

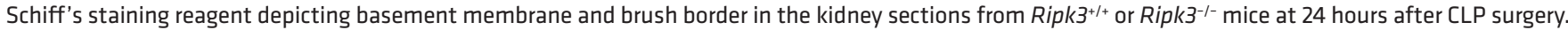
(C) Representative images showing KIM-1 immunohistochemical staining on the apical side of the tubular epithelial cells from Ripk $3^{+/+}$or Ripk $3^{-/-}$mice at 6 hours after CLP or sham surgery. (A-C) Scale bars: $20 \mu \mathrm{m}$ ( $n=3$ for Sham; $n=6$ for CLP). (D) Semiquantitative analysis of tubular injury (tubular atrophy or dilatation, loss of brush border, vacuolization, epithelial cell shedding, and denuded tubular basement membrane) scored as: 0 , normal; $1,<10 \% ; 2,10 \%$ $25 \% ; 3,25 \%-50 \% ; 4,50 \%-75 \% ; 5,75 \%-100 \%$ of affected area from 20 random fields. (E and $\mathbf{F}$ ) Urinary neutrophil gelatinase associated lipocalin-2 (E) and $\beta 2$-microglobulin (F) normalized to urine creatinine of Ripk $3^{+/+}$or $R i p k 3^{-1-}$ mice at 6 hours after CLP or sham surgery. Data are mean \pm SEM of 3 independent experiments ( $n=4$ mice/group). ${ }^{* *} P<0.01,{ }^{* *} P<0.001$, 1-way ANOVA.

intracellular vacuolization - a marker of kidney injury - was evident in the kidney tubular epithelial cells of Ripk $^{3^{-/}}$mice relative to Ripk $3^{+/+}$mice (Figure 2A). Furthermore, loss of brush border in the renal

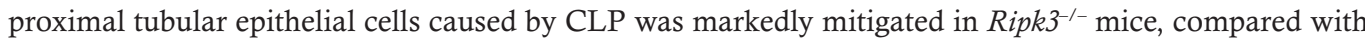
WT Ripk $3^{+/+}$mice (Figure 2B). Immunohistochemical analysis revealed increased staining of kidney injury molecule-1 (KIM-1) on the apical side of kidney tubular epithelial cells in kidney sections of Ripk $3^{+/+}$

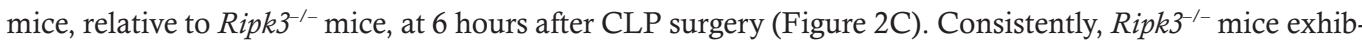
ited a markedly reduced kidney injury score relative to $R i p k 3^{+/+}$mice after CLP (Figure 2D). Interestingly, the ratio of urinary lipocalin-2 to urinary creatinine, a marker of kidney injury, was markedly elevated in $R i p k 3^{+/+}$mice at 6 hours after CLP relative to sham mice and was markedly lower in ipk $^{-/-}$mice relative to Ripk $^{+/+}$mice at 6 hours after CLP (Figure 2E). The ratio of urinary $\beta 2$-microglobulin to urinary creatinine, an indicator of kidney tubular injury, was also higher in Ripk $3^{+/+}$mice subjected to CLP relative to sham mice and was lower in CLP-treated Ripk $3^{-1-}$ mice relative to Ripk $3^{+/+}$mice (Figure 2F). Furthermore, blood urea nitrogen (BUN) levels, indicative of kidney dysfunction, were markedly decreased in $R i p k 3^{-/-}$mice relative to Ripk $3^{+/+}$mice subjected to CLP surgery, suggesting preserved kidney function in Ripk $3^{-1-}$ mice after CLP (Supplemental Figure 2A). The level of total urinary protein standardized to urine 
A
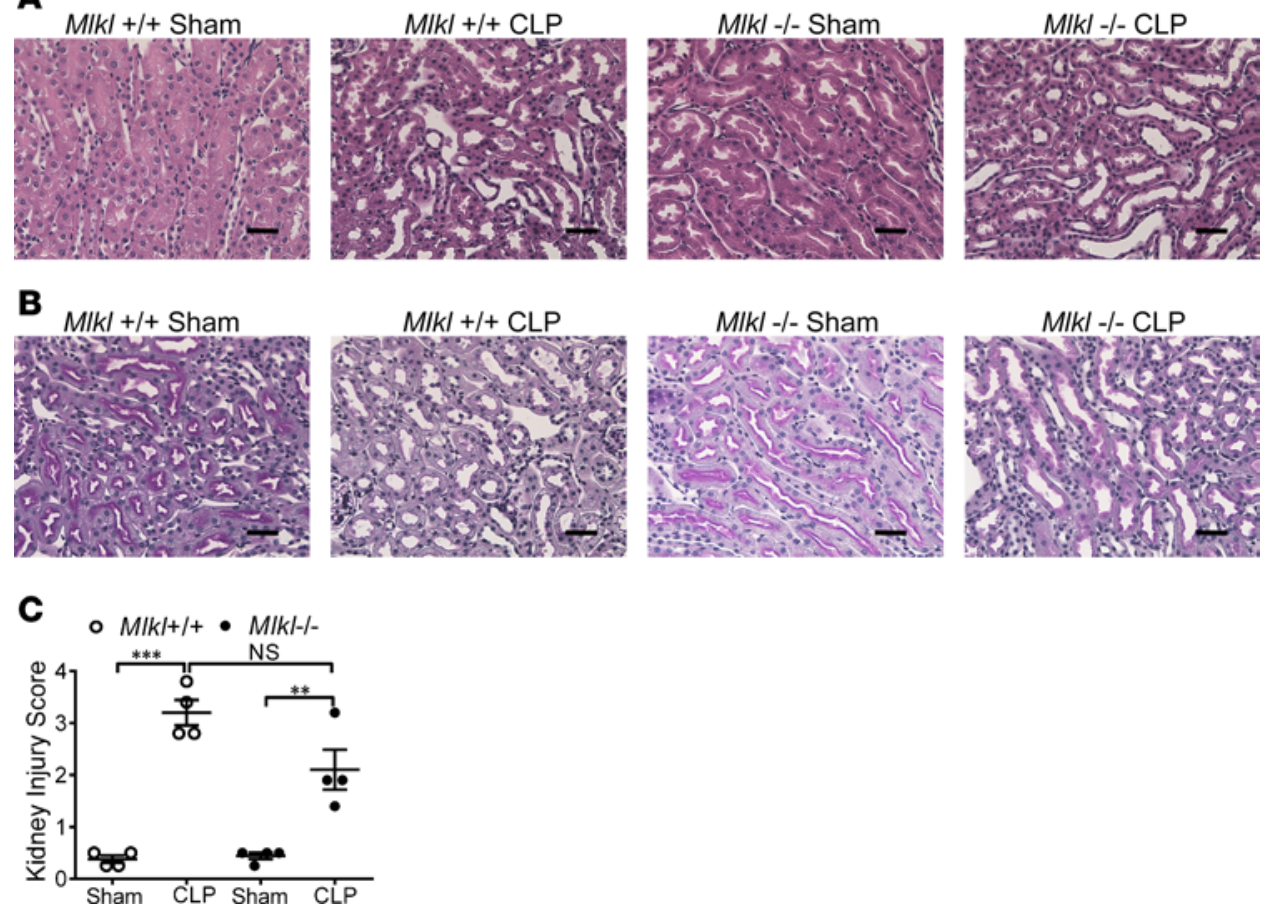

Figure 3. MIkl deficiency does not protect against kidney tubular injury in sepsis. (A) Representative images of H\&E staining of tubular epithelial cells of kidney sections from Mlkl+/+ or Mlkll- mice at 24 hours after CLP surgery. (B) Representative images of periodic acid Schiff's staining reagent

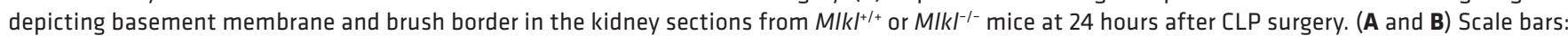
$20 \mu \mathrm{m}$ ( $n=4$ for Sham; $n=4$ for CLP). (C) Semiquantitative analysis of tubular injury (tubular atrophy or dilatation, loss of brush border, vacuolization, epithelial cell shedding, and denuded tubular basement membrane) scored as: 0, normal; $1,<10 \% ; 2,10 \%-25 \% ; 3,25 \%-50 \% ; 4,50 \%-75 \%$; $5,75 \%-100 \%$ of affected area from 20 random fields. Data are means \pm SEM of 3 independent experiments $\left(n=4\right.$ mice/group). ${ }^{* *} P<0.01,{ }^{* * *} P<$ 0.001, 1-way ANOVA with Tukey's post hoc test.

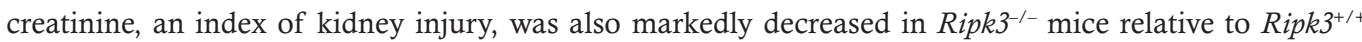
mice in response to CLP surgery (Supplemental Figure 2B).

Sepsis increases RIPK3-dependent cell death in kidney tubular epithelial cells. Since RIPK3 can signal to the necroptosis pathway, we examined the occurrence of cell death in kidney tissues of mice subjected to CLP. Although there remains no gold-standard assay for necroptosis, we used currently accepted methods, including necroptosis marker RIPK3 as described above - in combination with TUNEL staining, transmission electron microscopy, and use of a RIPK3-specific inhibitor - to assess necroptosis in kidney epithelial cells and tissue (13). We first investigated the incidence of necroptosis in kidney sections from Ripk3 ${ }^{-/-}$and $R i p k 3^{+/+}$mice subjected to CLP. We observed a low incidence of TUNEL-positive staining in the kidney tubular epithelial cells of Ripk $^{+/+}$mice subjected to CLP, relative to sham-operated mice, which was markedly higher than that observed in $R i p k 3^{-1-}$ mice (Supplemental Figure 3A).

Following LPS stimulation (24 hours), HK-2 cells that were pretreated with the RIPK3-specific inhibitor GSK' 872 (14) displayed markedly decreased LDH release, an indicator of membrane disruption and cytotoxicity, compared with vehicle-pretreated HK-2 cells (Supplemental Figure 3B). To examine whether genetic deficiency of RIPK3 could suppress kidney tubular epithelial cell death in vitro, we used RIPK3-targeted guide RNA (gRNA) to delete human RIPK3 in cas9-expressing HK-2 cells via CRISPR technology (Supplemental Figure 3C). LPS-treated HK-2 cells transfected with nontargeting (NT) gRNA were positive for ethidium homodimer-I (Ethd-I) staining, indicative of necrotic cell death with compromised membrane integrity. In contrast, HK-2 cells transfected with RIPK3 gRNA displayed markedly lower Ethd-I-positive staining relative to control cells at 24 hours following LPS challenge (Supplemental Figure 3D). We also examined LPS-induced cell death in HK-2 cells treated in the absence or presence of the apoptosis (pancaspase) inhibitor Z-VAD-FMK, or the RIPK1-specific necroptosis inhibitor necrostatin-1. Interestingly, both the apoptosis inhibitor and the necroptosis inhibitor markedly decreased LPS-induced cell death as compared with LPS treatment alone (Supplemental Figure 4). These results suggest that LPS-induced cell death in HK-2 cells involves components of both caspase-dependent apoptosis and necroptosis. 
A

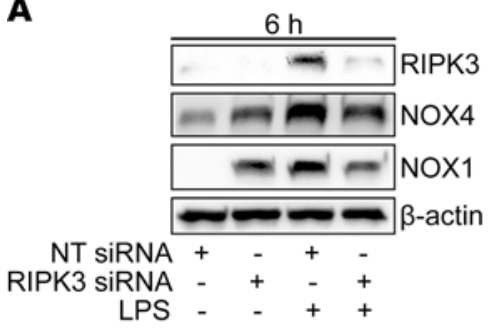

D

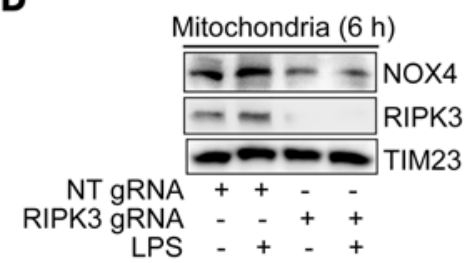

G

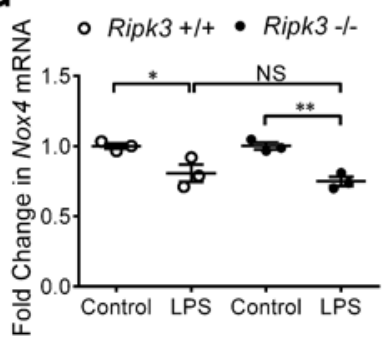

B

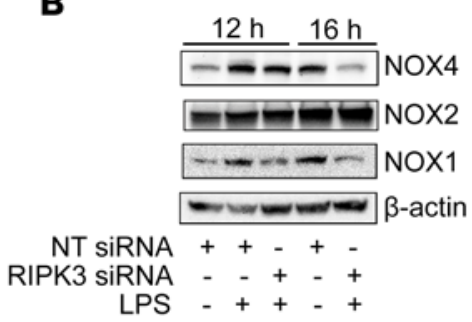

E

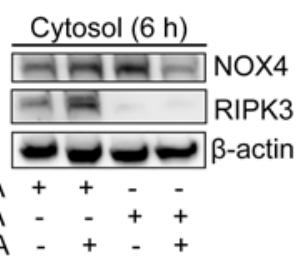

C

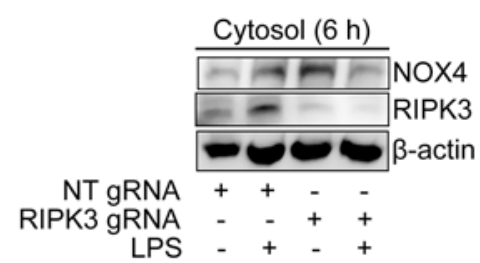

$\mathbf{F}$
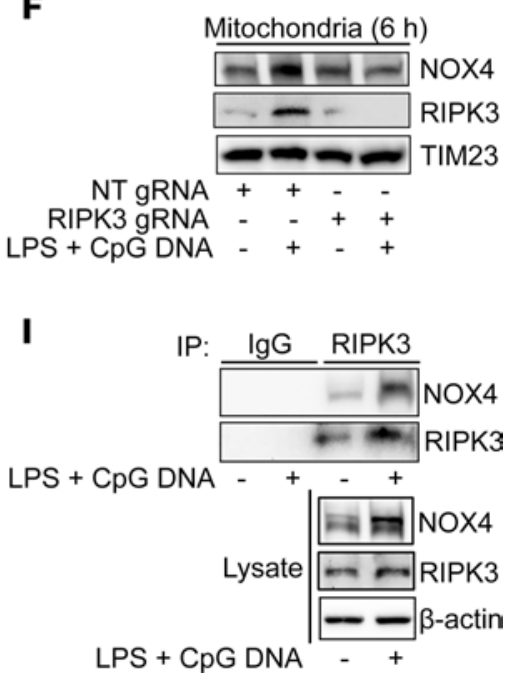

Figure 4. RIPK3 regulates NOX4 expression through posttranscriptional mechanism in vitro. (A) nontargeting (NT) gRNA- or RIPK3 gRNA-transduced HK-2 cells were incubated in the absence or presence of LPS $(1 \mu \mathrm{g} / \mathrm{ml})$ for indicated time interval. Western blot for the expression of RIPK3, NOX4, and NOX1. (B) HK-2 cells were transfected with RIPK3 siRNA or NT siRNA and then treated with LPS $(1 \mu \mathrm{g} / \mathrm{ml})$ for the indicated time intervals. Western blot for the expression of NOX4, NOX2, and NOX1. (C-F) Western blot for the expression of NOX4 and RIPK3 in the (C and E) cytosolic and (D and F) mitochondrial fractions of NT gRNAor RIPK3 gRNA-transduced HK-2 cells that were incubated in the absence or presence of LPS $(1 \mu \mathrm{g} / \mathrm{ml})$ (C and $\mathbf{D})$ or LPS + CpG DNA (1 $\mu \mathrm{g} / \mathrm{ml})(\mathbf{E}$ and $\mathbf{F})$ for indicated time interval. (G-H) Nox4 mRNA expression in the primary tubular epithelial cells obtained from Ripk $3^{+/+}$and Ripk $3^{-/-}$mice treated with (G) LPS (1 $\left.\mu \mathrm{g} / \mathrm{ml}\right)$ or (H) LPS + CpG DNA $(1 \mu \mathrm{g} / \mathrm{ml})$ at the indicated time interval. Data are means \pm SEM of 3 independent experiments. ${ }^{*} P<0.05,{ }^{* *} P<0.01,1$-way ANOVA with Tukey's post hoc test. (I) HK-2 cells were incubated in the absence or presence of LPS + CpG DNA $(1 \mu \mathrm{g} / \mathrm{ml})$ for 6 hours. Lysates were immunoprecipitated with IgC and RIPK3; then, the immunoprecipitates or control lysates were blotted for NOX4 or RIPK3. Data shown are representative of 3 independent experiments.

MLKL deficiency does not reduce kidney tubular injury in sepsis. MLKL, an interactive partner and downstream phosphorylation target of RIPK3, has been implicated as a primary effector of the RIPK3-dependent necroptosis pathway (6). We therefore investigated the functional role of MLKL in sepsis-induced AKI. We subjected mice with genetic deletion of $\operatorname{MLKL}\left(\mathrm{Mlkl}^{--}\right)$, and their corresponding WT $\left(M l k t^{++}\right)$mice, to CLP or sham surgery. In contrast to RIPK3 deficiency, genetic deletion of MLKL did not markedly protect mice from kidney tubular injury at 24 hours after CLP, as indicated by H\&E staining (Figure 3A), periodic acid Schiff's (PAS) staining (Figure 3B), and kidney injury scores (Figure 3C). To examine the functional role of MLKL in AKI, we measured BUN and urinary lipocalin-2 normalized to urine creatinine levels in $\mathrm{Mlkl}^{l^{++}}$ and $\mathrm{Mlkt}^{-1}$ mice at 6 hours after CLP or sham surgery. The BUN levels were not markedly different in the $\mathrm{Mlkt}^{\text {-- }}$ mice relative to $\mathrm{Mlkl}^{+/+}$mice subjected to CLP surgery (Supplemental Figure 5A). In contrast, the ratio of lipocalin-2 to urine creatinine levels was lower in $\mathrm{Mlkl}^{--}$mice as compared with WT mice subjected

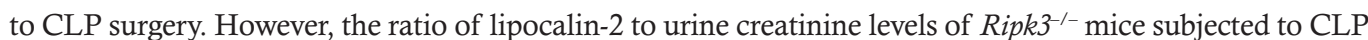
surgery was markedly lower compared with either WT or $\mathrm{Mlkl}^{--}$mice (Supplemental Figure 5B). These data are consistent with a dominant role for RIPK3 in sepsis-induced kidney dysfunction.

RIPK3 regulates NOX4 expression during sepsis-induced kidney injury. We have recently demonstrated that NOX4 is a critical regulator of macrophage inflammatory responses in vitro (15). The role of RIPK3 as a regulator of NOX isoforms in sepsis has not been previously studied. We therefore examined the role of RIPK3 in NOX regulation using NT gRNA- (control) or RIPK3 gRNA-transfected HK-2 cells challenged with LPS. The expression levels of NOX1 and NOX4 were lower in RIPK3-deficient HK-2 cells following 
A

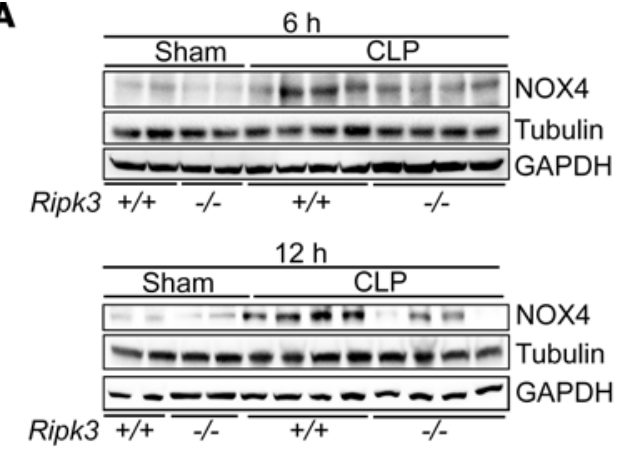

B

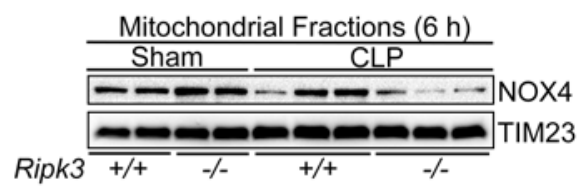

C

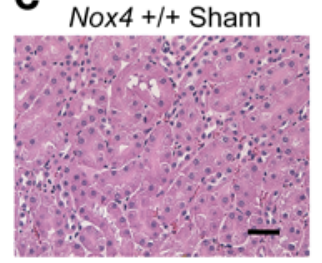

D

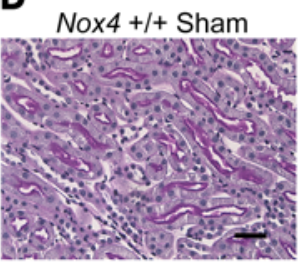

Nox $4+1+$ CLP

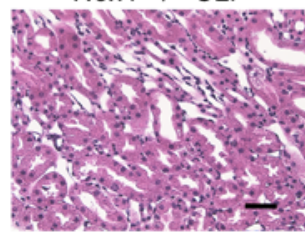

Nox4 +/+ CLP

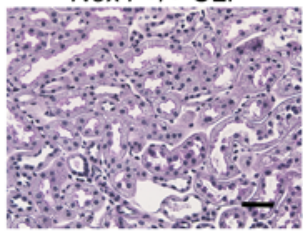

Nox4 -/- Sham

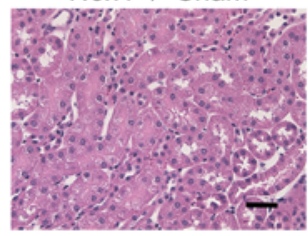

Nox4 -/- Sham

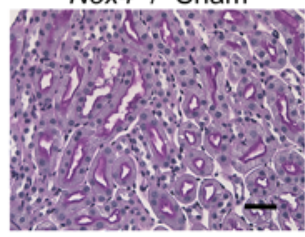

Nox4 -I- CLP
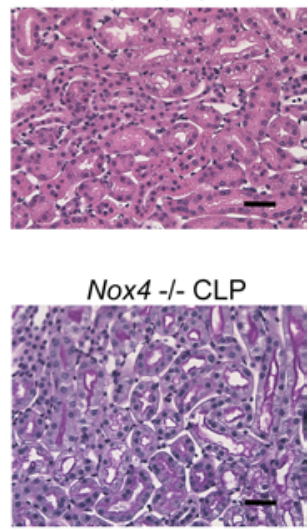

$\mathbf{E}$

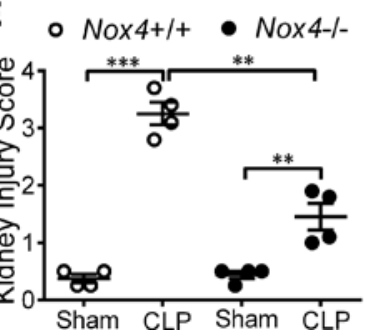

Figure 5. RIPK3 regulates NOX4 expression in vivo, and NOX4 deficiency decreases kidney tubular injury in sepsis. (A) Western blot for expression of NOX4 in the kidney homogenates of Ripk $3^{+/+}$or Ripk $3^{-/-}$mice at 6 hours (upper) and 12 hours (lower) after CLP or sham surgery ( $n=4$ mice/ group). (B) Western blot for NOX4 expression in the mitochondrial fractions of kidney homogenates of Ripk $3^{+/+}$or Ripk $3^{-1-}$ mice at 6 hours after CLP or sham surgery. Western blots shown are representative results from 3 independent experiments. (C) Representative images of H\&E staining of tubular epithelial cells of kidney sections from $\mathrm{Nox}_{4}^{+++}$or $\mathrm{Nox}^{-/-}$mice at 24 hours after CLP surgery. (D) Representative images of periodic acid Schiff's staining reagent depicting basement membrane

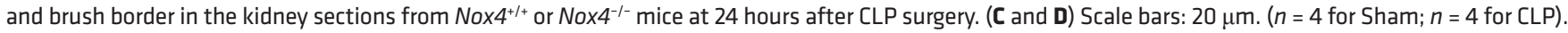
(E) Semiquantitative analysis of tubular injury (tubular atrophy or dilatation, loss of brush border, vacuolization, epithelial cell shedding, and denuded tubular basement membrane) scored as: 0, normal; 1, <10\%; 2, 10\%-25\%; 3, 25\%-50\%; 4, 50\%-75\%; 5, 75\%-100\% of affected area from 20 random fields. Data are means \pm SEM of 3 independent experiments ( $n=4$ mice/group). ${ }^{* *} P<0.01,{ }^{* *} P<0.001,1$-way ANOVA with Tukey's post hoc test.

LPS addition, compared with control HK-2 cells (Figure 4A). Similarly, we used RIPK3-specific siRNA to knock down RIPK3 expression in HK-2 cells prior to LPS challenge. As expected, the expression levels of NOX2, the phagocytic isoform of NADPH oxidase, were not altered, whereas those of NOX1 and NOX4 were lower in RIPK3 siRNA-transfected HK-2 cells at 12 hours and 16 hours following LPS treatment, relative to NT control siRNA-transfected HK-2 cells (Figure 4B).

We have previously observed that the expression of NOX4 is induced by proinflammatory stimuli and localized primarily to the mitochondria of macrophages (15). To further elucidate the mechanism by which RIPK3 can regulate NOX4, we isolated cytosolic and mitochondrial fractions (15) from NT gRNA- (control) or RIPK3 gRNA-transfected HK-2 cells that were challenged with LPS or with the combination of LPS and CpG bacterial DNA (16). Following cellular stimulation with either LPS (Figure 4, C and D) or LPS and CpG DNA (Figure 4, E and F), the expression of NOX4 was lower both in cytosolic (Figure 4, C and E) 

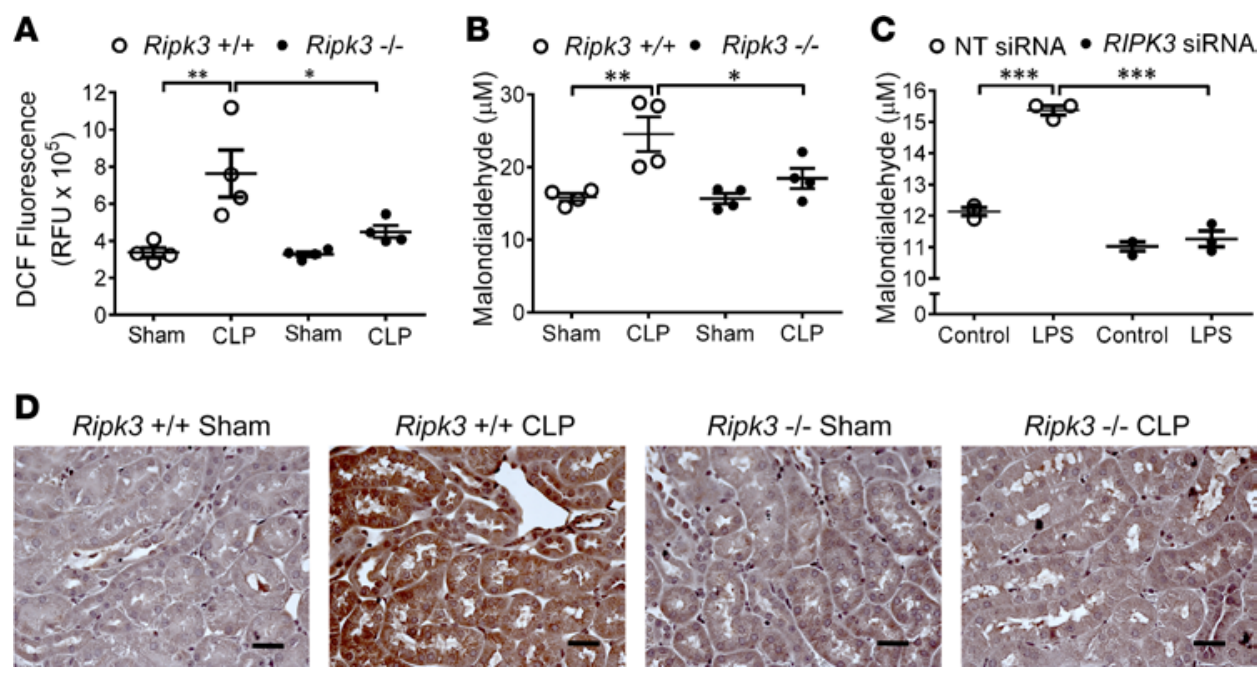

Figure 6. Ripk3 deficiency reduces oxidative stress in septic kidney. (A) ROS levels were determined by DCFDA conversion to DCF in the kidney homog-

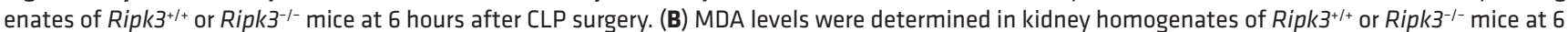
hours after CLP surgery. (C) HK-2 cell lysates were transfected with RIPK3 siRNA, followed by incubation in the absence or presence of LPS (1 $\mu \mathrm{g} / \mathrm{ml})$ for 6 hours. MDA levels were determined in cell lysates. (D) Representative IHC images of nitrotyrosine staining in the tubular epithelial cells of kidney sections from Ripk $3^{+/+}$or Ripk $3^{-/-}$mice at 12 hours after CLP or sham surgery. Scale bars: $20 \mu \mathrm{m}$ ( $n=4$ for Sham; $n=4$ for CLP). Data are means \pm SEM of 3 independent experiments ( $n=4$ mice/group). ${ }^{*} P<0.05,{ }^{* *} P<0.01,{ }^{* *} P<0.001,1$-way ANOVA with Tukey's post hoc test.

and mitochondrial fractions (Figure 4, D and F) isolated from RIPK3 gRNA-transfected HK-2 cells, relative to those isolated from NT gRNA-transfected cells. Interestingly, Nox4 mRNA expression was downregulated by LPS in primary tubular epithelial cells isolated from $R i p k 3^{+/+}$or $R i p k 3^{-/-}$mice, and the response did not

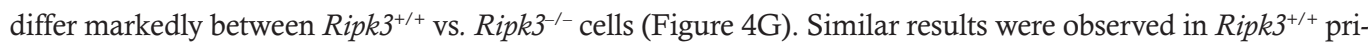
mary tubular epithelial cells challenged with CpG DNA, although the relative decrease in Nox4 expression observed in Ripk $3^{+/+}$cells was not significant in Ripk $3^{-/-}$cells (Figure $4 \mathrm{H}$ ). These data suggest that the regulation of NOX4 expression by RIPK3 may occur through a posttranscriptional mechanism. To investigate the possibility that RIPK3 may regulate NOX4 via protein-protein interaction, we performed coimmunoprecipitation experiments. We found that NOX4 protein coimmunoprecipitated with RIPK3 in whole cell lysates from HK-2 cells and that the expression of this complex was increased in cells challenged with LPS and CpG DNA (Figure 4I). These data, taken together, suggest that RIPK3 regulates the accumulation of NOX4 in both the cytosol and mitochondria in response to proinflammatory stimuli, through a posttranscriptional mechanism potentially involving intermolecular interactions between RIPK3 and NOX4.

Similar observations were made in vivo in mice subjected to CLP. Importantly, the elevated NOX4 expression in the kidney tissues of $R i p k 3^{+/+}$mice at 6 hours and 12 hours following CLP surgery relative to sham-operated mice was lower in $R i p k 3^{-1}$ mice (Figure 5A). We also found that the expression of NOX4 was higher in mitochondrial fractions isolated from kidney tissues of $R i p k 3^{+/+}$mice relative to $R i p k 3^{-/-}$mice at 6 hours following CLP surgery (Figure 5B). These data suggest that RIPK3 can regulate mitochondrial NOX4 expression in the context of sepsis-induced AKI.

NOX4 deficiency decreases kidney tubular injury in sepsis. We next sought to investigate the role of NOX4 in

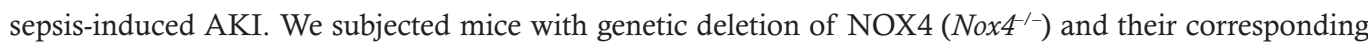
WT $\left(\mathrm{Nox}^{+/+}\right)$mice to CLP or sham surgery. Histological analysis by H\&E staining, PAS staining (Figure

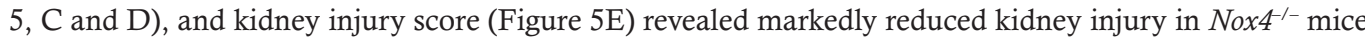
compared with $\mathrm{Nox}^{+/+}$mice at 24 hours following CLP.

RIPK3 deficiency decreases ROS levels in kidney during sepsis. We next investigated the role of RIPK3 in regulating ROS production in kidney tissue during sepsis. ROS levels, as assessed by 2',7'- dichlorofluorescein (DCF) fluorescence, were approximately 2-fold higher in kidney tissues of $R i p k 3^{+/+}$mice subjected to CLP (6 hours), relative to sham-operated Ripk $3^{+/+}$mice, and were markedly lower in the kidney tissues of Ripk $3^{-/-}$ mice subjected to CLP (Figure 6A). Consistent with these results, the levels of malondialdehyde (MDA), an indicator of lipid peroxidation, were also markedly lower in the kidney tissues of Ripk $3^{-1-}$ mice relative to Ripk $^{+/+}$mice after CLP surgery (Figure 6B). We also confirmed in cultured HK-2 cells that LPS treatment 
A

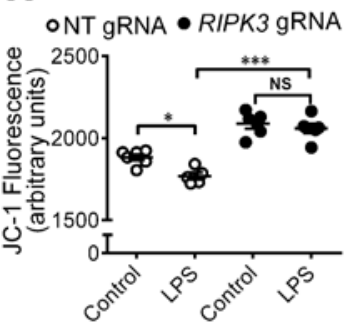

B

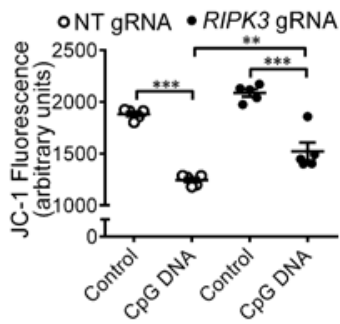

C

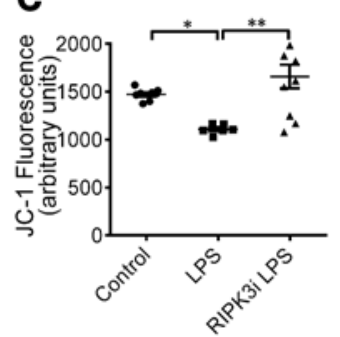

D

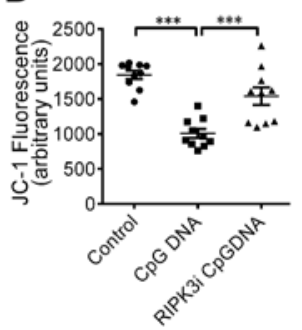

E

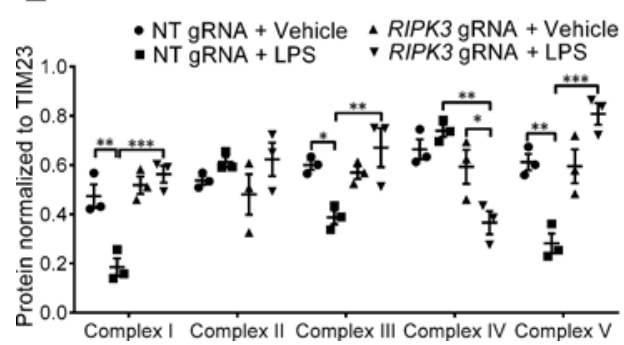

$\mathbf{F}$

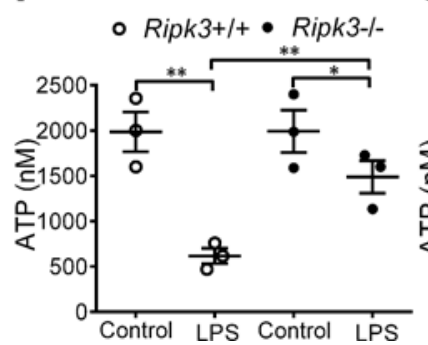

G

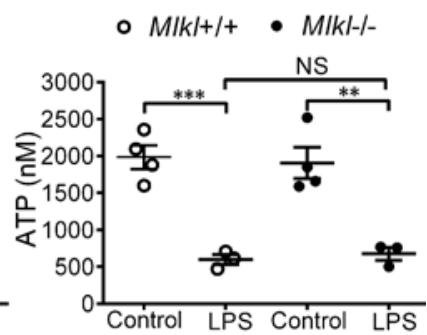

Figure 7. Ripk3 deficiency reduces mitochondrial dysfunction in vitro. (A and B) NT gRNA- or RIPK3 gRNA-transfected HK-2 cells were incubated in the absence or presence of LPS $(1 \mu \mathrm{g} / \mathrm{ml})$ or CpG DNA $(1 \mu \mathrm{g} / \mathrm{ml})$ for 24 hours. (C and D) HK-2 cells were pretreated with RIPK3 inhibitor (CSK'872) (1 $\mu$ M) and then incubated in the absence or presence of LPS $(1 \mu \mathrm{g} / \mathrm{ml})$ or CpG DNA $(1 \mu \mathrm{g} / \mathrm{ml})$. JC-1 fluorescence was determined at 24 hours. (E) NT gRNA- or RIPK3 gRNAtransfected HK-2 cells were treated with or without LPS $(1 \mu \mathrm{g} / \mathrm{ml})$ and mitochondrial fractions were isolated at 6 hours. Protein levels of mitochondrial complex I (NDUFB8), -II (SDHB), -III (UQCRC-1), -IV (MTC01), and -V (ATP5A) subunit levels (normalized to TIM23) were quantified in the mitochondrial fractions.

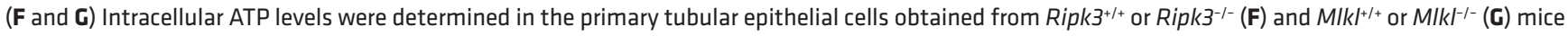
treated with LPS at 6 hours. Data are means \pm SEM of 3 independent experiments ( $n=4$ mice/group). Data are means \pm SEM of 3 independent experiments. ${ }^{*} P<0.05,{ }^{* *} P<0.01,{ }^{* *} P<0.001,2$-way ANOVA with Bonferroni's post-hoc test (E) or 1-way ANOVA with Tukey's post-hoc test (A-D, F, and G).

caused an increase in MDA levels in NT siRNA-transfected cells but not in Ripk3 siRNA-transfected cells (Figure 6C). Using IHC, we observed lower nitrotyrosine expression - a marker of nitrosative stress - in kidneys from $R i p k 3^{-/-}$mice, relative to $R i p k 3^{+/+}$mice 6 hours after CLP (Figure 6D). These results indicate that RIPK3 promotes oxidative/nitrosative stress in vitro and in vivo in the context of sepsis.

RIPK3 deficiency decreases mitochondrial dysfunction in vitro. To study the role of RIPK3 in mitochondrial dysfunction, we treated HK-2 cells with LPS or CpG/bacterial DNA, which causes loss of mitochondrial membrane potential $(\triangle \Psi \mathrm{m})(16)$. RIPK3-deficient (RIPK3 gRNA-transfected) HK-2 cells displayed markedly improved $\triangle \Psi \mathrm{m}$, as assessed by JC-1 fluorescence, relative to NT gRNA-transfected HK-2 cells 24 hours after treatment with LPS or CpG DNA (Figure 7, A and B). Similarly, treatment with the RIPK3 inhibitor GSK'872 rescued HK-2 cells from loss of $\triangle \Psi \mathrm{m}$ induced by LPS or CpG DNA (Figure 7, C and D). To determine the impact of RIPK3 on mitochondrial respiratory chain complexes, we isolated mitochondrial fractions from RIPK3 gRNA-transfected HK-2 cells following LPS exposure. Quantitative analysis of Western blots demonstrated that LPS treatment reduced the expression of mitochondrial complex I, -III, and -V in NT gRNAtransfected HK-2 cells but not in RIPK3 gRNA-transfected HK-2 cells (Figure 7E). To assess the functional relevance of this finding, we also measured intracellular ATP levels following LPS treatment in primary kidney tubular epithelial cell cultures obtained from $R i p k 3^{+/+}$and $R i p k 3^{-/}$mice. Exposure to LPS markedly lowered ATP levels in Ripk $3^{+/+}$kidney tubular epithelial cells, relative to vehicle-treated cells. However, the reduction of ATP levels following LPS treatment was markedly less in Ripk3 ${ }^{-1}$ kidney tubular epithelial cells (Figure 7F). In contrast, we found no significant difference in the reduction of ATP levels following LPS treatment in primary tubular epithelial cells obtained from $\mathrm{Mlkl}^{l_{++}}$and $\mathrm{Mlkl}^{\mathrm{H}^{--}}$mice (Figure 7G).

RIPK3 deficiency suppresses mitochondrial dysfunction during sepsis-induced kidney injury. We next evaluated mitochondrial injury in kidney tubular epithelial cells of mice subjected to sepsis-induced AKI. To evaluate mitochondrial morphology, we performed transmission electron microscopy on kidney sections harvested from Ripk $3^{-1-}$ and $R i p k 3^{+/+}$mice subjected to CLP. We observed in vivo evidence of mitochondrial injury, as indicated by ultrastructural changes (i.e., widening of mitochondrial cristae and increased swelling of mitochondria) in the kidney tubular epithelial cells of $R i p k 3^{+/+}$mice after CLP; this was not evident in Ripk $^{-1-}$ mice (Figure 8, A and B). 
A

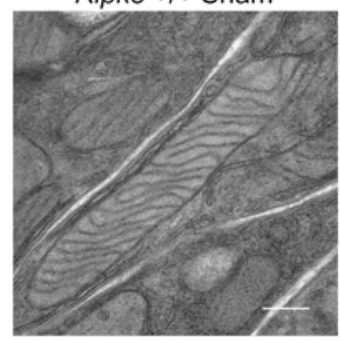

B

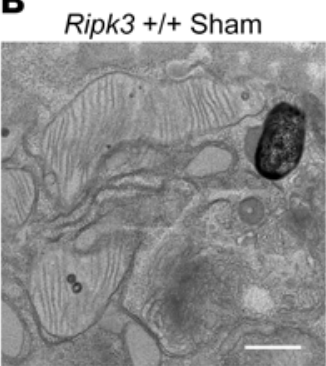

Ripk3 +/+ Sham

Ripk3 +/+ CLP

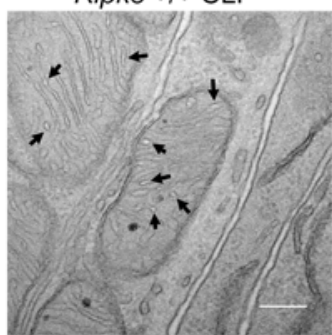

Ripk3 +/+ CLP

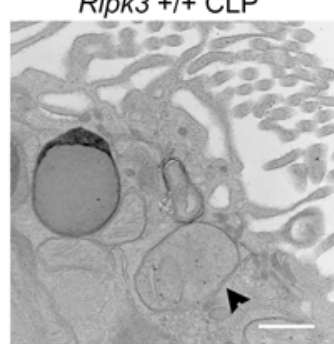

Ripk3+/+ Sham ^ Ripk3-/-Sham 口 Ripk3+/+ CLP VRipk3-/- CLP

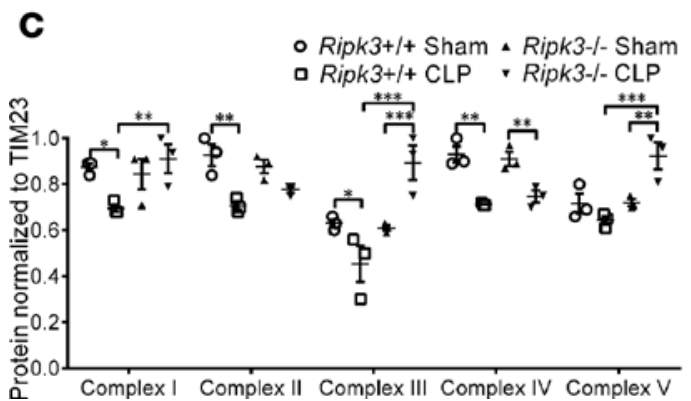

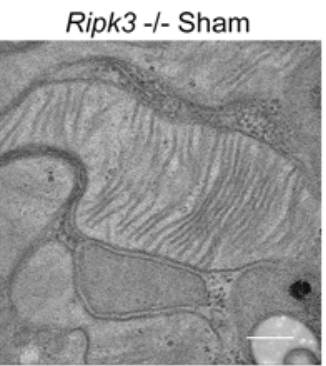

Ripk3 -/- Sham

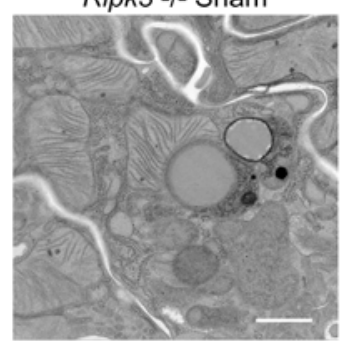

D

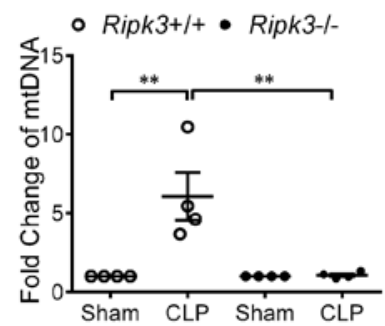

Ripk3 -/- CLP

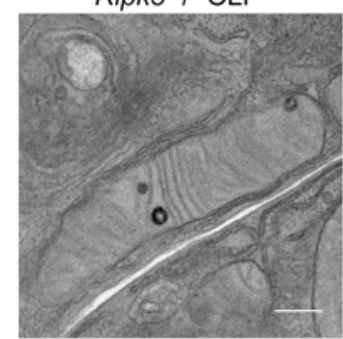

Ripk3 -/- CLP

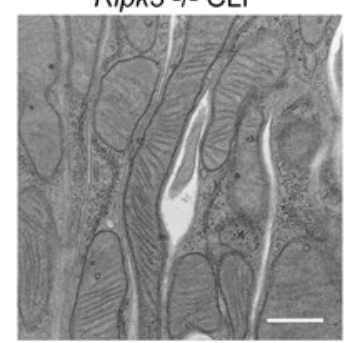

E

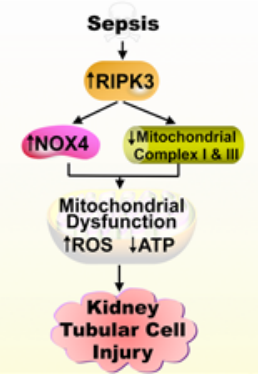

Figure 8. Ripk3 deficiency reduces mitochondrial dysfunction in vivo. (A) Representative transmission electron microscopy images displaying mitochon-

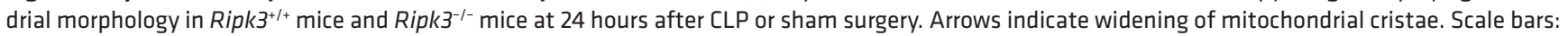
$200 \mathrm{~nm}$. (B) Representative transmission electron microscopy images of tubular epithelial cells of kidney sections from Ripk $3^{+/+}$or Ripk $3^{-/-}$mice at 24 hours after CLP surgery. Arrowhead indicates swelling of mitochondria. Scale bars: $500 \mathrm{~nm}$. (A and B) Four to 5 fields/image were analyzed, $20-25$ images per animal ( $n=4$ for Sham; $n=4$ for CLP). (C) Protein quantification of mitochondrial complex I (NDUFB8), -II (SDHB), -III (UQCRC-I), -IV (MTCO1), and -V

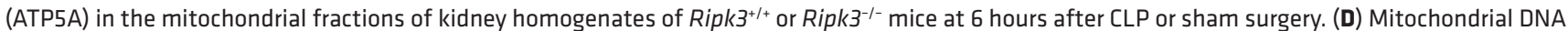

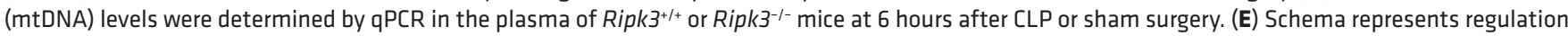
of sepsis-induced mitochondrial dysfunction and necroptosis in the proximal tubules of the kidney. Data are means \pm SEM of 3 independent experiments ( $n=4$ mice/group). ${ }^{*} P<0.05,{ }^{* *} P<0.01,{ }^{* * *} P<0.001,2$-way ANOVA with Bonferroni's post-hoc test (C) or 1-way ANOVA with Tukey's post-hoc test (D).

To further examine if RIPK3 contributes to changes in mitochondrial complex protein expression

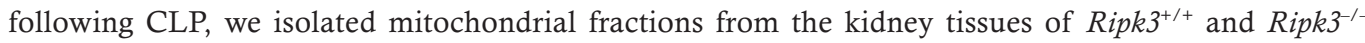
mice. Interestingly, we observed decreases in the expression of mitochondrial complex I and -III in Ripk $3^{+/+}$mice following CLP, but not in $\mathrm{Ripk}^{-/-}$mice (Figure 8C). These findings demonstrate that RIPK3 regulates the expression of mitochondrial complex I and -III in sepsis-induced kidney injury. Additionally, elevated mitochondrial DNA (mtDNA) levels, a marker of mitochondrial injury, were observed in the plasma of Ripk $^{+/+}$mice 6 hours after CLP, relative to sham control; this was not observed in ipk $^{3^{-1}}$ mice (Figure 8D).

RIPK3 expression and mtDNA are increased in the plasma and urine of patients with sepsis. To translate our in vitro and in vivo findings to human disease, we measured RIPK3 mRNA expression in the urinary cells and RIPK3 protein expression in the urine of patients admitted to the intensive care unit (ICU) with sepsis. We observed markedly higher RIPK3 (mRNA and protein) levels in patients with sepsis compared with patients without sepsis (Figure 9, A and B, and Supplemental Figure 6, A and B). Moreover, RIPK3 protein expression was markedly higher in both urine and plasma samples from patients with sepsis and AKI compared with patients without AKI (Figure 9, C and D). We have previously shown that plasma mtDNA levels are correlated with mortality in critically ill patients (17). Higher mtDNA copy number, as determined 

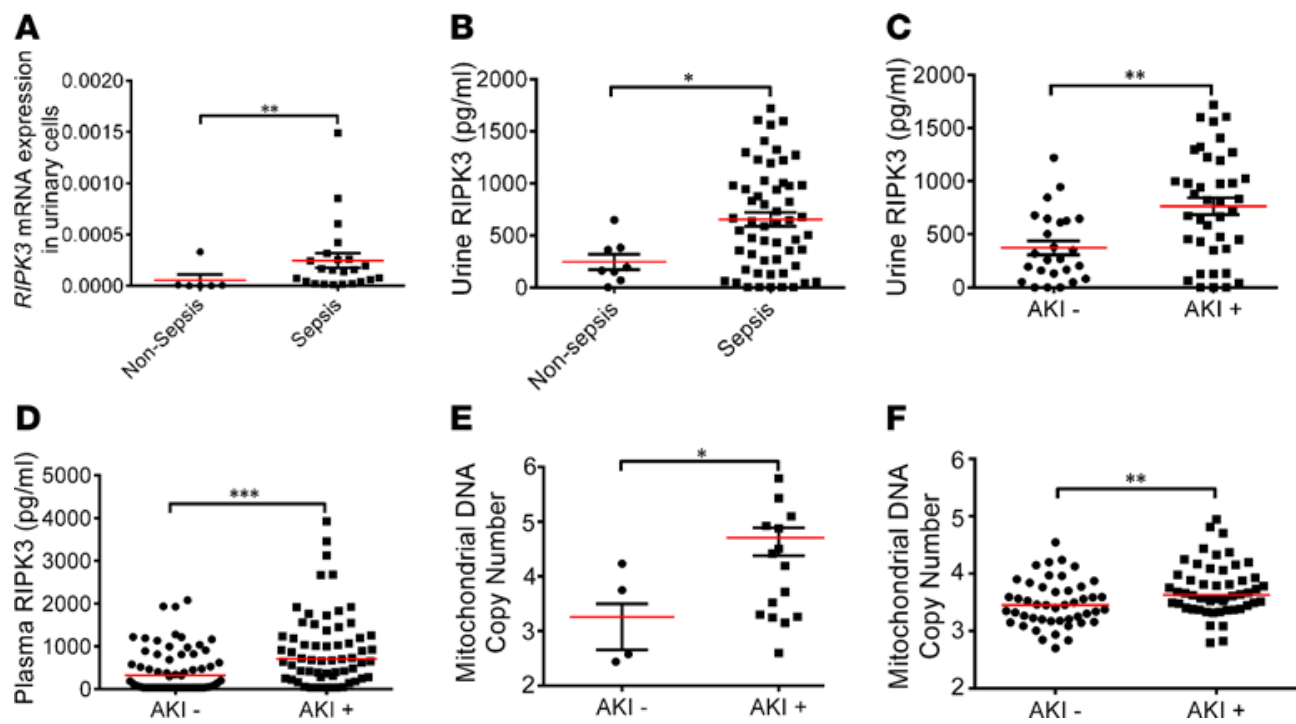

Figure 9. RIPK3 and mitochondrial DNA levels are increased in human sepsis/AKI patients. (A) RIPK3 mRNA expression was determined by qPCR in the human urinary cells of nonsepsis patients $(n=6)$ compared with sepsis patients $(n=23)$. (B) RIPK3 protein expression was determined by ELISA in the human urine supernatant samples of nonsepsis patients $(n=8)$ compared with sepsis patients $(n=55)$. (C and D) RIPK3 protein expression in the human urine (C) and plasma (D) samples of sepsis-induced AKI patients ( $n=36$ for urine; $n=61$ for plasma) versus non-AKI patients ( $n=23$ for urine; $n=59$ for plasma). (E and F) mtDNA (MT-ND1 expression) was determined by qPCR in the human urine (E) and plasma (F) samples of non-AKI patients $(n=4$ for urine; $n=47$ for plasma) versus sepsis-induced AKI patients ( $n=15$ for urine; $n=52$ for plasma). mtDNA copy number was expressed in log units. Data are mean \pm SEM. Red bars represent mean. ${ }^{*} P<0.05,{ }^{* *} P<0.01$, ${ }^{* *} P<0.001$, student's unpaired 2-tailed $t$ test.

by quantitative PCR (qPCR) detection of mitochondrially encoded NADH dehydrogenase 1 (MT-NDI), was detected in the urine and plasma samples from patients with sepsis and AKI compared with patients without AKI (Figure 9, E and F). These results indicate that urine and plasma RIPK3 protein levels, as well as mtDNA copy number, are markedly associated with sepsis-induced AKI.

In contrast, the mRNA expression levels of RIPK1 and MLKL in the urinary cells of sepsis patients were not markedly different from those of nonsepsis patients (Supplemental Figure 7, A and D). The expression levels of RIPK1 (Supplemental Figure 7, B and C) and MLKL (Supplemental Figure 7, E and F) in either the urine or plasma of sepsis patients were not markedly different from those of nonsepsis patients.

\section{Discussion}

Mitochondrial dysfunction has been increasingly recognized as a key contributor to the pathogenesis of human diseases $(15,17,18)$. Our findings provide strong evidence, both in vitro and in vivo, that in sepsis-induced AKI, RIPK3 promotes kidney tubular injury via mitochondrial dysfunction, involving the increased mitochondrial localization of NOX4 and the inhibition of mitochondrial complex I and -III. RIPK3 and related signaling molecules (e.g., RIPK1 and MLKL) may exert pleiotropic cellular functions, including but not limited to the activation of the necroptosis signaling pathway $(6,7,19)$ and the modulation of inflammation (20).

In the current study, we hypothesized that inhibiting the RIPK3-dependent signaling pathway may confer renal protection in sepsis. Consistently, we demonstrate that genetic deficiency of Ripk3 protected against tissue injury, loss of kidney function, and mitochondrial dysfunction in the kidney tubular epithelial cells of mice subjected to polymicrobial sepsis. Furthermore, we observed that elevated RIPK3 levels, but not RIPK1 or MLKL levels, in both urine and plasma were associated with AKI in patients with sepsis. Taken together, our data provide strong evidence that RIPK3-dependent mitochondrial dysfunction, leading to compromised kidney tubular epithelial cell function, may represent a key underlying mechanism in the pathogenesis of sepsis-induced AKI.

Previous studies have demonstrated the occurrence of both tubular necrosis and apoptosis in sepsis-induced AKI $(21,22)$. The study of Takasu et al. presented histological evidence for a low incidence of tubular necrosis in sepsis, with a rare instance of apoptosis in kidney tissue as detected by staining for 
cleaved cytokeratin-18 (23). Similar to the findings of Takasu et al., we have also observed a low incidence of necrotic cell death in kidney tubular epithelial cells of mice subjected to CLP.

RIPK3-mediated necroptosis has been suggested to depend on the availability and activation of pseu-

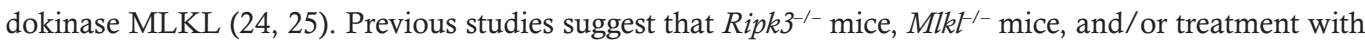
the necroptosis inhibitor necrostatin-1 were protective in cisplatin-induced AKI (26). However, increasing evidence suggests that RIPK3 and MLKL do not always act in tandem, such that their respective genet-

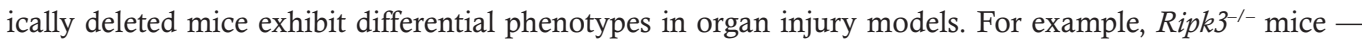
but not $\mathrm{Mlkt}^{-1-}$ mice - showed improved outcome in models of kidney ischemia/reperfusion injury and inflammation (27). Furthermore, RIPK3 - but not MLKL — was implicated in cardiomyocyte necroptosis induced by ischemia/reperfusion injury (28). In our studies, we found that, in contrast to the renopro-

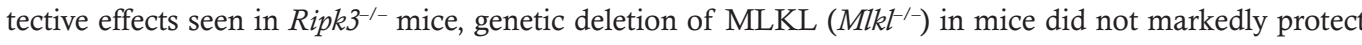
against kidney injury following CLP. Our findings therefore suggest that RIPK3 exerts effects on kidney function independently of MLKL. Interestingly, a recent report conversely showed that $M l k t^{-1-}$ mice, but not Ripk $^{-/-}$mice, were protected from experimental hepatitis (29). Importantly, MLKL-independent functions of RIPK3 have been previously reported. For example, in a mouse model of inflammatory arthritis, RIPK3 promoted IL-1 $\beta$ activation in an MLKL-independent fashion, indicating a potential role for RIPK3 in the regulation of inflammation (30).

Recently, we have demonstrated that NOX4 is a crucial signaling molecule for NLRP3 inflammasome activation in macrophages subjected to proinflammatory stimuli and may contribute to susceptibility to pneumonia infection (15). Previous studies have shown that NOX activity is increased in the peritoneal macrophages of mice subjected to CLP (31). Increased kidney NOX4 expression has been reported in diabetic nephropathy (32). However, the role of NOX4 in the propagation of sepsis-induced AKI and its relationship to RIPK3 has previously not been explored.

In the current study, we demonstrate that NOX4 expression was increased in kidney tubular epithelial cells both in vitro following LPS treatment and in vivo in the CLP-induced sepsis model. Using genetic interference or deletion studies, we demonstrate that RIPK3 is required for both the enhanced expression and mitochondrial accumulation of NOX4 in vitro and in vivo in response to proinflammatory stimuli. We have also found that the RIPK3-dependent regulation of NOX4 and its mitochondrial translocation likely occur via a posttranscriptional mechanism that involves increased intermolecular interaction between RIPK3 and NOX4. Taken together, our findings that RIPK3 regulates NOX4 expression and its mitochon-

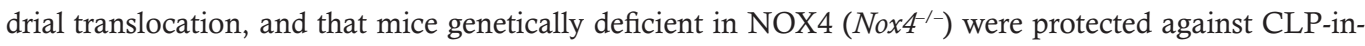
duced kidney tubular injury in a similar manner as Ripk3 ${ }^{-/-}$mice, suggest that RIPK3-dependent regulation of NOX4 expression plays a critical role in sepsis-induced AKI.

The progression of sepsis-induced AKI is characterized by a complex series of events involving both oxidative stress and mitochondrial dysfunction (18, 33-35). Consistently, we observed elevated markers of oxidative/nitrosative stress in kidney tubular epithelial cells in vitro following LPS treatment and in vivo following CLP, and we observed that RIPK3 deficiency abrogated these effects, suggesting that RIPK3 promotes sepsis-induced oxidative stress. We also demonstrate here that RIPK3 promotes mitochondrial dysfunction in sepsis-induced kidney injury. We found that RIPK3 regulates NOX4 expression in the mitochondria and that expression levels of mitochondrial complex I and -III were decreased in sepsis, in a manner dependent on RIPK3 expression. Our in vitro findings also suggest that RIPK3-dependent signaling contributes to mitochondrial depolarization and reduced expression of mitochondrial complex subunits in response to LPS. Consistently, genetic deletion of Ripk3 preserves cellular ATP production in primary tubular epithelial cells following LPS treatment. Taken together, our results suggest that RIPK3 promotes mitochondrial dysfunction by upregulating NOX4 and by downregulating the expression levels of mitochondrial complex I and -III subunits during sepsis-induced AKI. We conclude that the effects of RIPK3 leading to compromised epithelial cell function are primarily dependent on mitochondrial functional alterations and are independent of MLKL.

A previous report has suggested that plasma RIPK3 expression is associated with AKI in critically ill trauma patients (12). Our clinical findings identify an association between elevated levels of RIPK3 (mRNA and protein) expression in urinary cells and urine of patients with sepsis. However, the expression levels of RIPK1 and MLKL both in plasma and urine were not markedly different in sepsis patients compared with nonsepsis patients. To our knowledge, this is the first study to demonstrate urinary RIPK3 as a biomarker of sepsis-induced AKI. The utilization of urinary biomarkers might be a sensitive, noninvasive, 
and clinically useful method that is particularly attractive, given that urine is an easily available source. Our results also indicate that both urinary and plasma RIPK3 levels are associated with AKI among patients with sepsis. In addition, we also demonstrate increased mtDNA copy number in the urine and plasma of patients with sepsis-induced AKI. While we have previously reported that plasma mtDNA levels correlated with mortality in critically ill patients (17), our current study is the first demonstration to our knowledge of urinary mtDNA associated with sepsis-induced AKI.

Taken together, our studies identify a RIPK3-dependent mitochondrial pathway as a potential therapeutic target in sepsis-induced AKI (Figure 8E) and identifies that urinary and plasma levels of RIPK3 may serve as novel biomarkers of human sepsis-induced AKI.

\section{Methods}

Chemicals and reagents. The chemicals used in the experiments included bacterial LPS (catalog tlrl-3pelps; InvivoGen), CpG DNA (ODN 2395, catalog tlrl-2395; InvivoGen), and chemical inhibitors RIPK3 inhibitor (GSK'872, catalog 530389; MilliporeSigma) and NOX4 inhibitor (catalog S7171; Selleck Chemicals). All other chemicals were reagent grade (MilliporeSigma).

Cell Culture. HK-2 cells (catalog CRL-2190; ATCC) were maintained in DMEM supplemented with $10 \% \mathrm{v} / \mathrm{v}$ FBS (Invitrogen), $100 \mathrm{U} / \mathrm{ml}$ penicillin, and $100 \mu \mathrm{g} / \mathrm{ml}$ streptomycin in humidified incubators at $37^{\circ} \mathrm{C}$ in a $95 \%$ air $-5 \% \mathrm{CO}_{2}$ atmosphere.

Animals. C57BL/6N mice were purchased from Charles River Laboratories. Mice with targeted deletion of Ripk3 $3^{-1-}$ on a C57BL/6N background were obtained from the laboratory of Vishva Dixit (Genentech). These mice have been characterized previously (36). $M l k l^{-1}$ mice were provided by the laboratory

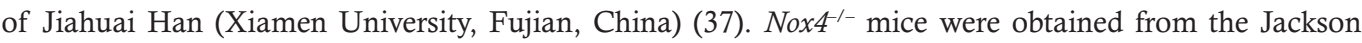
laboratory and were generated as described previously (38).

CLP mouse model. All mice aged 8 weeks old were subjected to the CLP procedure and allowed to recover for the indicated time points as previously described (39). Briefly, $60 \%$ of the cecum was ligated to achieve a midgrade CLP. Using a 21-gauge needle, the ligated cecum was perforated in a through-and-through puncture fashion. The ligated cecum was gently squeezed to extrude a small amount of feces from either side of the perforation sites. The ligated cecum was then returned into the peritoneal cavity; then, the abdominal musculature was closed using surgical sutures. Throughout the experiments, mice were given food and water ad libitum.

Isolation of murine primary tubular kidney epithelial cells. Primary tubular epithelial cells were isolated from $R i p k 3^{+/+}$and $R i p k 3^{-/-}$mice as previously described (40). Briefly, kidney cortices were dissected from Ripk $3^{-1-}$ mice and their WT Ripk $3^{+/+}$littermates and minced with a sterile scalpel. The minced portion was filtered through a 70- $\mu \mathrm{m}$ cell strainer with media (Renal Epithelial Cell Growth Medium; PromoCell). The medium containing tubules and other cells were plated on collagen-coated culture plates overnight at $37^{\circ} \mathrm{C}$ in $5 \% \mathrm{CO}_{2}$. On day 2, the medium containing the tubules was transferred to a conical tube, centrifuged at $130 \mathrm{~g}$ for 5 minutes, plated on collagen-coated culture plates, and then incubated for 72 hours at $37^{\circ} \mathrm{C}$ in $5 \% \mathrm{CO}_{2}$. Each experiment required tubular epithelial cells generated from a single animal, and cells were not passaged.

CRISPR/Cas9 genome editing and screening. HK-2 cells were stably transduced with Blast-Cas9 lentivirus (catalog VCAS10126; GE Healthcare). gRNA fragments targeting the human RIPK3 gene were designed and transfected into Cas9 expressing HK-2 cells. Upon overnight transfection with the above gRNAs (RIPK3 gRNA, CATGGAGAACGGCTCCTTGT), the transfected cells were seeded at low density $(1 \times$ $10^{3}$ cells $/ 10-\mathrm{cm}$ dish) to obtain independent clonal lines. The colonies were screened by PCR and followed by Sanger sequencing. Cell lines with double-stranded breaks for RIPK3 gene were identified and assayed for lactate dehydrogenase and JC-1 dye activities.

siRNA transfection. Human SMARTpool on-target plus RIPK3-specific siRNAs were purchased from Dharmacon (GE Healthcare). HK-2 cells were first transfected with respective siRNAs using transfection reagent (Lipofectamine RNAimax; Thermo Fisher Scientific). Then, HK-2 cells were subjected to LPS addition. Next, cells were lysed for protein extraction, and samples were analyzed by Western blotting.

$R N A$ isolation and $q P C R$. Total RNA was extracted from whole kidney tissues using RNeasy Mini Kit (catalog 74104; Qiagen Inc.) according to the manufacturer's instructions. cDNA was prepared using the standard protocol (catalog 1754555; Invitrogen). The qPCR were performed on a 7500 Fast real-time PCR system (Applied Biosystems) using 0.2 $\mu \mathrm{M}$ Ripk3 primers (catalog 4331182, ID: Mm00444947_m1; Qiagen Inc.). $\mathrm{Ct}$ values were determined. Forty cycles of amplification were performed. The gene of interest was normalized to the $\mathrm{Ct}$ value of the endogenous reference gene, GAPDH, using the $\triangle \mathrm{Ct}$ method. 
Isolation of cytosolic and mitochondrial fractions. Cytosolic and mitochondrial fractions were isolated from cells using a mitochondria/cytosol fraction kit (K265-25, Biovision) according to the manufacturer's instructions. The cytosolic $\beta$-actin and mitochondrial inner membrane protein Tim 23 served as standards for cytosol and mitochondrial protein, respectively (41).

Western blotting. HK-2 cells and mouse kidney tissues were harvested in M-PER (catalog 78501; Thermo Fisher Scientific) and T-PER (catalog 78510; Thermo Fisher Scientific) lysis buffer. Protein concentrations were determined using the BCA assay (catalog 23225; Thermo Fisher Scientific). Protein extracts (both cellular and tissue homogenates) were subjected to SDS-PAGE separation on a Mini-Protean TGX gel electrophoretic system (Bio-Rad). Proteins were electroblotted onto PVDF membranes (catalog 162-0177; Bio-Rad). After transfer, nonspecific binding was prevented using 5\% skimmed milk TBS-Tween (Santa Cruz Biotechnology Inc.). After blocking, the membranes were incubated with rabbit polyclonal or monoclonal antibodies. The antibodies used for Western blotting were anti-mouse RIPK3 (catalog 2283; Prosci), anti-human RIPK3 (catalog ab152130; Abcam), anti-human p-RIPK3 (S227) (catalog ab209384; Abcam), anti-mouse p-RIPK3 (S232) (catalog ab195117; Abcam), anti-human RIPK1 (catalog 3493S; Cell Signaling Technologies), anti-human p-MLKL (catalog 91689; Cell Signaling Technologies), anti-mouse MLKL (catalog MABC604; MilliporeSigma), anti-human NOX4 (catalog ab133303; Abcam), anti-human NOX2 (catalog ab129068; Abcam), anti-human NOX1 (catalog ab131088; Abcam), anti-mouse nitrotyrosine (catalog ab5532; MilliporeSigma), anti-human or -mouse ACTB/ $\beta$-actin (catalog sc-47778; Santa Cruz Biotechnology Inc.), anti-human or -mouse tubulin (catalog 2125; Cell Signaling Technologies). Chemiluminescent bands were visualized using enhanced chemiluminescence solution (Thermo Fisher Scientific). Quantification of Western blots was performed using ImageJ software version 1.46 (NIH).

Immunoprecipitation. HK-2 cells were harvested after treatment with LPS and CpG DNA for 6 hours. The cells were washed and collected in ice-cold PBS and pelleted by centrifugation at $800 \mathrm{~g}$ for 5 minutes at $4^{\circ} \mathrm{C}$. The cell pellet was resuspended in IP lysis buffer (catalog 14321D; Thermo Fisher Scientific) and incubated for 15 minutes on ice. The samples were centrifuged at $700 \mathrm{~g}$ for 10 minutes at $4^{\circ} \mathrm{C}$ to eliminate nuclear fractions and further centrifuged at $13,000 \mathrm{~g}$ for 30 minutes at $4^{\circ} \mathrm{C}$ to obtain cytosolic fractions. The cytosolic fractions were applied to dynabeads prebound with RIPK3 antibody (catalog 13526; Cell Signaling Technology) and immunoprecipitated with gentle mixing for 2 hours at $4^{\circ} \mathrm{C}$. The immunoprecipitated proteins were eluted and subjected to immunoblotting as recommended by the manufacturer's instructions (catalog 14321D; Thermo Fisher Scientific).

Measurement of ROS generation in kidney homogenates. ROS levels in the kidney tissues or HK-2 cell homogenates were assayed using 2',7'-dichlorodihydrofluorescein diacetate (DCFDA) as previously described (42). Briefly, freshly prepared protein lysates were diluted 1:20 with ice-cold Locke's buffer ( $\mathrm{pH}$

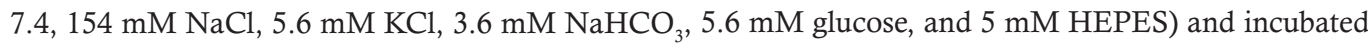
with DCFDA. The formation of DCF was assayed (485/535 nm, excitation/emission [ex/em]) using a fluorometer (Glomax Multi+ microplate reader, Promega).

Measurement of MDA levels. Following CLP surgery, whole kidney tissues or HK-2 cell homogenates were extracted and protein samples were harvested. MDA, a compound produced during lipid peroxidation, was measured using a commercial assay kit (catalog 700870; Cayman Chemical) based on the reaction with thiobarbituric acid.

Measurement of $\Delta \Psi m$. The $\Delta \Psi \mathrm{m}$ was measured using a JC-1 dye (catalog 551302; BD Biosciences) according to the manufacturer's instructions. Briefly, $2 \times 10^{3} \mathrm{HK}-2$ cells/well were seeded into black-bottomed 96-well optical plates (Greiner Bio-One). After overnight cell seeding, the cells were treated with LPS and CpG for 24 hours. JC-1-stained cells were measured (488/590 nm, ex/em) using a fluorometer (Glomax Multi+ microplate reader, Promega).

Measurement of cell viability and cell death. Cell viability and cell death assays were measured using fluorescent dyes (catalog G9201; Promega) according to manufacturer's instructions. Briefly, $1 \times 10^{4} \mathrm{HK}-2$ cells/well were seeded into black-bottomed 96-well optical plates (Greiner Bio-One). After overnight cell seeding, the cells were treated with LPS for 24 hours. Cell viability and cell death were measured using a fluorometer (Glomax Multi+ microplate reader, Promega).

ATP assay. The amounts of intracellular ATP in the primary tubular epithelial cells from Ripk $3^{+/+}$ and Ripk $3^{-1-}$ mice was measured according to the manufacturer's instructions (catalog 700410; Cayman Chemical). Briefly, primary tubular epithelial cells were grown in serum-free conditions overnight and then incubated in the presence or absence of LPS for 6 hours. The primary tubular epithelial cells 
were trypsinized, and an equal number of cells were taken and lysed to release the intracellular ATP. The released ATP was measured using a luciferin/luciferase assay using a luminometer (Promega Glo $\mathrm{Max}+$, Promega).

Histology. Whole kidney tissues obtained from mice challenged with CLP or sham surgery were fixed overnight in 4\% PFA. After washing in fresh PBS, fixed tissues were dehydrated, cleared, and embedded in paraffin by routine methods. Sections $(5 \mu \mathrm{m})$ were collected on Superfrost Plus positively charged microscope slides (Thermo Fisher Scientific), deparaffinized, and stained with H\&E and PAS reagents.

Assessment of kidney injury score. Kidney injury score is scored as: 0 , normal; $1,<10 \% ; 2,10 \%-25 \% ; 3$, $25 \%-50 \% ; 4,50 \%-75 \% ; 5,75 \%-100 \%$ of affected kidney areas obtained from WT and Ripk3-/- Mlkt $^{-1-}$, and $\mathrm{Nox}^{-/-}$mice subjected to sham or CLP surgery. This criteria includes tubular atrophy or dilatation, loss of brush border, vacuolization, epithelial cell shedding, and denuded tubular basement membrane.

IHC. IHC staining was performed on 4\% paraformaldehyde fixed paraffin-embedded samples using standard protocols as previously described (43). Briefly, 5- $\mu$ m paraffin tissue sections were subjected to steam boiling (catalog IW-1102; IHC World) for 30 minutes in antigen retrieval buffer ( $\mathrm{pH}$ 6.0) and allowed to cool at room temperature for 20 minutes. Sections were quenched in $30 \%$ hydrogen peroxide at room temperature for 20 minutes. Then, slides were incubated with blocking buffer, followed by Avidin D and Biotin blocking reagent (Vector Laboratories). Kidney sections were stained with anti-RIPK3 antibody (catalog 2283; ProSci) or KIM-1 antibody (catalog ab78494; Abcam) and incubated overnight at $4^{\circ} \mathrm{C}$. Sections were developed with Vectastain ABC kit (catalogPK4001; Vector Laboratories) followed by hematoxylin staining (catalogH3401; Vector Laboratories).

Transmission electron microscopy. Kidney specimens were fixed with $2.5 \%$ glutaraldehyde, $4 \%$ paraformaldehyde, and $0.02 \%$ picric acid in $0.1 \mathrm{M}$ sodium cacodylate buffer at $\mathrm{pH} 7.2$. Following a secondary fixation in $1 \%$ osmium tetroxide and $1.5 \%$ potassium ferrocyanide, the samples were dehydrated through a graded ethanol series and embedded in an epon analog resin. Ultrathin sections were cut using Accu-Edge high-profile blades (catalog 4685; Sakura) on a Microm HM 355S (Thermo Fisher Scientific). Sections were collected on copper grids, further contrasted with lead citrate, and viewed on a JEM 1400 electron microscope (JEOL). Images were recorded with a Veleta $2 \mathrm{~K}$ digital camera (Olympus-SIS).

TUNEL assay. TUNEL assay was performed according to the manufacturers' instructions (catalog 4810-30-K; R\&D Systems). Briefly, paraffin embedded, 4\% paraformaldehyde fixed mouse kidney sections were deparaffinized and rehydrated. TUNEL-positive nuclei were analyzed using a microscope equipped with digital camera (EVOS, Invitrogen). Ten to 12 randomly selected fields were counted.

Taqman PCR. Total RNA was extracted either from murine tubular epithelial cells or from urinary cell pellets of all human subjects using RNeasy mini kit (catalog 74104; Qiagen) according to the manufacturer's instructions. Samples that had an RNA purity $\left(\mathrm{A}_{260}: \mathrm{A}_{280}\right.$ ratio) greater than or equal to 1.8 were considered in the present study. Nox4 mRNA expression (catalog 4448892; ID: Mm00479246_m1; Thermo Fisher Scientific) in the murine tubular epithelial cells or RIPK1 mRNA expression (catalog 4448892; ID: Hs01041870_m1), or RIPK3 mRNA expression or MLKL mRNA expression (catalog 4448892; ID: Hs00414923_m1), in the urinary cell pellets of critically ill patients was determined using the quantitative Taqman PCR method. The data were normalized to a housekeeping gene 18s rRNA (catalog 4333760T; Thermo Fisher Scientific) for human samples and to Gapdh (catalog 4331182; ID: Mm99999915_g1; Thermo Fisher Scientific) for mouse samples. Human RIPK3 primers for Taqman PCR were listed in Table 1. qPCR was performed in the ABI PRISM 7500 Real-Time PCR System (Applied Biosystems).

ELISA. Protein expression levels of RIPK1 (catalog LS-F9254; LSBio), RIPK3 (catalog CSB-EL019737HU; Cusabio), and MLKL (catalog LS-F18428; LSBio) in the urine and plasma of critically ill patients was determined by an ELISA kit according to the manufacturer's instructions. Lipocalin-2 (catalog MLCN20; R\&D Biosystems) and 32 -microglobulin (catalog ABIN773404; Antibodies-Online) protein expression in the urine from $R i p k 3^{+/+}$and $R i p k 3^{-1-}$ mice subjected to CLP were determined by ELISA kits according to the manufacturer's instructions. Total protein, BUN, and creatinine in the urine or serum from $R i p k 3^{+/+}$and $R i p k 3^{-/-}$mice were determined by using Beckman Coulter chemistry analyzer AU680.

SYBR green PCR for MT-ND1 expression ( $m t D N A$ ) in human samples. Total genomic DNA was extracted from all human urine and plasma samples using DNeasy blood and tissue kit (catalog 69506; Qiagen) 
Table 1. The primer sequences used in the present study for qPCR system

\begin{tabular}{|c|c|}
\hline Name of the gene & Sequence (5' to $\left.3^{\prime}\right)$ \\
\hline \multirow[b]{2}{*}{ huRIPK3 } & Forward, GAAGGACTGAAGGAGCTAATCCA \\
\hline & Reverse, TTTTGGTAGGCATTCCTGGAA \\
\hline \multirow{2}{*}{ huMT - ND1 } & Forward, ATACCCATGGCCAACCTC \\
\hline & Reverse, GGGCCTTTGCGTAGTTGTAT \\
\hline
\end{tabular}

according to the manufacturer's instructions. Samples that had a DNA purity $\left(\mathrm{A}_{260}: \mathrm{A}_{280}\right.$ ratio $)$ greater than or equal to 1.8 were considered in the present study. Human mtDNA expression in the urine and plasma was determined using the quantitative SYBR green PCR method. Human MT-ND1 primers for SYBR green PCR are listed in Table 1. qPCR was performed in the ABI PRISM 7500 Real-Time PCR System (Applied Biosystems).

Human urine, urinary cells, and plasma sample collection. The Weill Cornell Medicine Registry and Biobank of Critically Ill Patients was initiated in 10/2014 as an ongoing prospective cohort of critically ill adult patients admitted to the New York Presbyterian Hospital Weill Cornell Medical Center. All patients admitted to the medical ICU are eligible for enrollment in the Registry and Biobank, unless they are cognitively impaired, are unable to provide informed consent (or an appropriate legal representative cannot not be found to provide consent), are admitted to the hospital purely to facilitate comfort care, are unwilling to receive blood transfusion, or present with a hemoglobin level of $<7 \mathrm{~g} / \mathrm{dl}$ upon admission. Finally, only patients with suspicion of infection were considered for inclusion in the present study. Biological samples (plasma, urine, and urinary cells), along with extensive clinical information (including vital signs before and during ICU hospitalization), are prospectively collected by physicians. Biological control samples refer to patients with critical illness, without evidence of sepsis. The baseline characteristics of all human subjects in the present study are listed in Supplemental Table 1.

Statistics. Values are expressed as mean \pm SEM. Groups were compared with the Student's 2-tailed unpaired $t$ test or 1-way ANOVA (followed by Tukey's multiple comparison post-hoc test) or 2-way ANOVA as appropriate (followed by Bonferroni's multiple comparison post-hoc test), using GraphPad Prism 6.0. A value of $P<0.05$ was considered statistically significant.

Study approvals. All animal experiments were performed under animal study protocols approved by the IACUC of Weill Cornell Medicine. The human subjects protocol was approved by the Weill Cornell Medicine IRB. Written informed consent was obtained from all human subjects.

\section{Author contributions}

$\mathrm{AS}, \mathrm{AMKC}$, and MEC designed the project; AS performed experiments with assistance from EP, KCM, EJF, KL, LG, and TM; AS, AMKC, and MEC analyzed the data and wrote the manuscript; OA and SWR reviewed and contributed to the writing of the manuscript. MEC supervised the project.

\section{Acknowledgments}

We thank I. Siempos (Weill Cornell Medical College) and E. Sanchez (Weill Cornell Medical College) for technical assistance. This work was supported in part by the NIH grants R01 DK57661 to MEC and R01 HL060234 and R01 HL133801 to MEC and AMKC.

Address correspondence to: Mary E. Choi, Division of Nephrology and Hypertension, Joan and Sanford I. Weill Department of Medicine, Weill Cornell Medical College, 525 East 68th Street, Box 3, New York, New York 10065, USA. Phone: 646.962.2605; Email: mechoi@med.cornell.edu. Augustine M. K. Choi, Division of Pulmonary and Critical Care Medicine, Joan and Sanford I. Weill Department of Medicine, Weill Cornell Medical College, 525 East 68th Street, Box 83, New York, New York 10065, USA. USA. Phone: 212.746.6005; Email: amchoi@med.cornell.edu. 
1. Singer M, et al. The Third International Consensus Definitions for Sepsis and Septic Shock (Sepsis-3). JAMA. 2016;315(8):801-810.

2. Rhodes A, et al. Surviving Sepsis Campaign: International Guidelines for Management of Sepsis and Septic Shock: 2016. Crit Care Med. 2017;45(3):486-552.

3. Gotts JE, Matthay MA. Sepsis: pathophysiology and clinical management. BMJ. 2016;353:11585.

4. Zarjou A, Agarwal A. Sepsis and acute kidney injury. J Am Soc Nephrol. 2011;22(6):999-1006.

5. Zarbock A, Gomez H, Kellum JA. Sepsis-induced acute kidney injury revisited: pathophysiology, prevention and future therapies. Curr Opin Crit Care. 2014;20(6):588-595.

6. Linkermann A, Green DR. Necroptosis. NEngl J Med. 2014;370(5):455-465.

7. Pasparakis M, Vandenabeele P. Necroptosis and its role in inflammation. Nature. 2015;517(7534):311-320.

8. Kitur K, et al. Necroptosis Promotes Staphylococcus aureus Clearance by Inhibiting Excessive Inflammatory Signaling. Cell Rep. 2016;16(8):2219-2230.

9. Moriwaki K, Bertin J, Gough PJ, Chan FK. A RIPK3-caspase 8 complex mediates atypical pro-IL-1 $\beta$ processing. J Immunol. 2015;194(4):1938-1944.

10. Linkermann A, et al. Synchronized renal tubular cell death involves ferroptosis. Proc Natl Acad Sci USA. 2014;111(47):16836-16841.

11. Qing DY, et al. Red blood cells induce necroptosis of lung endothelial cells and increase susceptibility to lung inflammation. Am J Respir Crit Care Med. 2014;190(11):1243-1254.

12. Shashaty MG, et al. Plasma Levels of Receptor Interacting Protein Kinase-3 (RIP3), an Essential Mediator of Necroptosis, are Associated with Acute Kidney Injury in Critically Ill Trauma Patients. Shock. 2016;46(2):139-143.

13. Ofengeim D, Yuan J. Regulation of RIP1 kinase signalling at the crossroads of inflammation and cell death. Nat Rev Mol Cell Biol. 2013;14(11):727-736.

14. Kaiser WJ, et al. Toll-like receptor 3-mediated necrosis via TRIF, RIP3, and MLKL. J Biol Chem. 2013;288(43):31268-31279.

15. Moon JS, et al. NOX4-dependent fatty acid oxidation promotes NLRP3 inflammasome activation in macrophages. Nat Med. 2016;22(9):1002-1012.

16. Yi AK, Yoon H, Park JE, Kim BS, Kim HJ, Martinez-Hernandez A. CpG DNA-mediated induction of acute liver injury in D-galactosamine-sensitized mice: the mitochondrial apoptotic pathway-dependent death of hepatocytes. J Biol Chem. 2006;281(21):15001-15012.

17. Nakahira K, et al. Circulating mitochondrial DNA in patients in the ICU as a marker of mortality: derivation and validation. PLoS Med. 2013;10(12):e1001577.

18. Galley HF. Oxidative stress and mitochondrial dysfunction in sepsis. Br J Anaesth. 2011;107(1):57-64.

19. Newton K, et al. Activity of protein kinase RIPK3 determines whether cells die by necroptosis or apoptosis. Science. 2014;343(6177):1357-1360.

20. Weinlich R, Oberst A, Beere HM, Green DR. Necroptosis in development, inflammation and disease. Nat Rev Mol Cell Biol. 2017;18(2):127-136.

21. Lerolle N, et al. Histopathology of septic shock induced acute kidney injury: apoptosis and leukocytic infiltration. Intensive Care Med. 2010;36(3):471-478.

22. Rameshkumar R, et al. Histopathological changes in septic acute kidney injury in critically ill children: a cohort of post-mortem renal biopsies. Clin Exp Nephrol. 2017;21(6):1075-1082.

23. Takasu O, et al. Mechanisms of cardiac and renal dysfunction in patients dying of sepsis. Am J Respir Crit Care Med. 2013;187(5):509-517.

24. Vanden Berghe T, Linkermann A, Jouan-Lanhouet S, Walczak H, Vandenabeele P. Regulated necrosis: the expanding network of non-apoptotic cell death pathways. Nat Rev Mol Cell Biol. 2014;15(2):135-147.

25. Mulay SR, et al. Cytotoxicity of crystals involves RIPK3-MLKL-mediated necroptosis. Nat Commun. 2016;7:10274

26. Xu Y, et al. A Role for Tubular Necroptosis in Cisplatin-Induced AKI. J Am Soc Nephrol. 2015;26(11):2647-2658.

27. Newton K, et al. RIPK3 deficiency or catalytically inactive RIPK1 provides greater benefit than MLKL deficiency in mouse models of inflammation and tissue injury. Cell Death Differ. 2016;23(9):1565-1576.

28. Zhang T, et al. CaMKII is a RIP3 substrate mediating ischemia- and oxidative stress-induced myocardial necroptosis. Nat Med. 2016;22(2):175-182.

29. Günther C, et al. The pseudokinase MLKL mediates programmed hepatocellular necrosis independently of RIPK3 during hepatitis. J Clin Invest. 2016;126(11):4346-4360.

30. Lawlor KE, et al. RIPK3 promotes cell death and NLRP3 inflammasome activation in the absence of MLKL. Nat Commun. $2015 ; 6: 6282$.

31. Zhang Z, et al. Plumbagin protects mice from lethal sepsis by modulating immunometabolism upstream of PKM2. Mol Med. 2016;22:162-172.

32. Sedeek M, et al. Critical role of Nox4-based NADPH oxidase in glucose-induced oxidative stress in the kidney: implications in type 2 diabetic nephropathy. Am J Physiol Renal Physiol. 2010;299(6):F1348-F1358.

33. Parikh SM. Therapeutic targeting of the mitochondrial dysfunction in septic acute kidney injury. Curr Opin Crit Care. 2013;19(6):554-559

34. Quoilin C, Mouithys-Mickalad A, Lécart S, Fontaine-Aupart MP, Hoebeke M. Evidence of oxidative stress and mitochondrial respiratory chain dysfunction in an in vitro model of sepsis-induced kidney injury. Biochim Biophys Acta. 2014;1837(10):1790-1800.

35. Brealey D, et al. Mitochondrial dysfunction in a long-term rodent model of sepsis and organ failure. Am J Physiol Regul Integr Comp Physiol. 2004;286(3):R491-R497.

36. Newton K, Sun X, Dixit VM. Kinase RIP3 is dispensable for normal NF-kappa Bs, signaling by the B-cell and T-cell receptors, tumor necrosis factor receptor 1, and Toll-like receptors 2 and 4. Mol Cell Biol. 2004;24(4):1464-1469.

37. Wu J, et al. Mlkl knockout mice demonstrate the indispensable role of Mlkl in necroptosis. Cell Res. 2013;23(8):994-1006.

38. Carnesecchi S, et al. A key role for NOX4 in epithelial cell death during development of lung fibrosis. Antioxid Redox Signal. 2011;15(3):607-619. 
39. Siempos II, Lam HC, Ding Y, Choi ME, Choi AM, Ryter SW. Cecal ligation and puncture-induced sepsis as a model to study autophagy in mice. $J$ Vis Exp. 2014;(84):e51066

40. Zarjou A, et al. Proximal tubule H-ferritin mediates iron trafficking in acute kidney injury. J Clin Invest. 2013;123(10):4423-4434.

41. Grohm J, et al. Inhibition of Drp1 provides neuroprotection in vitro and in vivo. Cell Death Differ. 2012;19(9):1446-1458

42. Ajay AK, Kim TM, Ramirez-Gonzalez V, Park PJ, Frank DA, Vaidya VS. A bioinformatics approach identifies signal transducer and activator of transcription-3 and checkpoint kinase 1 as upstream regulators of kidney injury molecule-1 after kidney injury. J Am Soc Nephrol. 2014;25(1):105-118.

43. Sureshbabu A, et al. Inhibition of Regulatory-Associated Protein of Mechanistic Target of Rapamycin Prevents Hyperoxia-Induced Lung Injury by Enhancing Autophagy and Reducing Apoptosis in Neonatal Mice. Am J Respir Cell Mol Biol. 2016;55(5):722-735 